\title{
The Impact of Power Allocation on Cooperative Non- orthogonal Multiple Access Networks With SWIPT
}

DOI:

10.1109/TWC.2017.2697380

\section{Document Version}

Accepted author manuscript

Link to publication record in Manchester Research Explorer

\section{Citation for published version (APA):}

Yang, Z., Ding, Z., Fan, P., \& Al-Dhahir, N. (2017). The Impact of Power Allocation on Cooperative Non-orthogonal Multiple Access Networks With SWIPT. Wireless Communications, IEEE Transactions on.

https://doi.org/10.1109/TWC.2017.2697380

\section{Published in:}

Wireless Communications, IEEE Transactions on

\section{Citing this paper}

Please note that where the full-text provided on Manchester Research Explorer is the Author Accepted Manuscript or Proof version this may differ from the final Published version. If citing, it is advised that you check and use the publisher's definitive version.

\section{General rights}

Copyright and moral rights for the publications made accessible in the Research Explorer are retained by the authors and/or other copyright owners and it is a condition of accessing publications that users recognise and abide by the legal requirements associated with these rights.

\section{Takedown policy}

If you believe that this document breaches copyright please refer to the University of Manchester's Takedown Procedures [http://man.ac.uk/04Y6Bo] or contact uml.scholarlycommunications@manchester.ac.uk providing relevant details, so we can investigate your claim.

\section{OPEN ACCESS}




\title{
The Impact of Power Allocation on Cooperative Non-orthogonal Multiple Access Networks with SWIPT
}

\author{
Zheng Yang, Zhiguo Ding, Senior Member, IEEE, Pingzhi Fan, Fellow, IEEE \\ and Naofal Al-Dhahir, Fellow, IEEE
}

\begin{abstract}
In this paper, a cooperative non-orthogonal multiple access (NOMA) network is considered, where a source communicates with two users through an energy harvesting relay. The impact of two types of NOMA power allocation policies, namely NOMA with fixed power allocation (F-NOMA) and cognitive radio inspired NOMA (CR-NOMA), on the considered cooperative simultaneous wireless information and power transfer (SWIPT) system is investigated. Particularly, closed-form expressions for the outage probability and their high SNR approximations are derived to characterize the performance of SWIPT-F-NOMA and SWIPT-CR-NOMA. These developed analytical results demonstrate that the two power allocation policies realize different tradeoffs between the reception reliability, user fairness and system complexity. Compared to conventional SWIPT relaying networks with orthogonal multiple access (OMA), the proposed NOMA schemes can effectively reduce the outage probability, although all of them realize the same diversity gain.
\end{abstract}

Index Terms-Non-orthogonal multiple access, simultaneous wireless information and power transfer, cognitive radio, outage probability, diversity gain.

\section{INTRODUCTION}

Non-orthogonal multiple access (NOMA) has recently attracted lots of attention, since it can provide high spectral efficiency and realize massive connectivity in the fifth generation (5G) mobile communication network [1] and [2]. In addition, a two-user special case of downlink NOMA has been adopted by Third Generation Partnership Project (3GPP) Long Term Evolution (LTE), termed multi-user superposition transmission (MUST) [3]. Unlike conventional orthogonal multiple access (OMA), multiple users in a NOMA network are allowed to share the same orthogonal resources in the time, frequency, and code domains. Meanwhile, successive interference cancellation (SIC) is applied at the receiver side,

Z. Yang is with the Institute of Mobile Communications, Southwest Jiaotong University, Chengdu 610031, China, and also with the College of Photonic and Electronic Engineering, Fujian Normal University, Fuzhou 350007, China. (email: zyfjnu@163.com).

Z. Ding is with the School of Computing and Communications, Lancaster University, LA1 4YW, UK. (e-mail: z.ding@lancaster.ac.uk).

P. Fan is with the Institute of Mobile Communications, Southwest Jiaotong University, Chengdu 610031, China. (email: p.fan@ieee.org).

N. Al-Dhahir is with the Department of Electrical Engineering, University of Texas at Dallas, Richardson, TX 75080 USA. (e-mail: aldhahir@utdallas.edu).

The work of Zheng Yang and Pingzhi Fan was supported by the National Science and Technology Major Project under Grant 2016ZX03001018-002, the Huawei HIRP Project under Grant YB201504, and the 111 Project under Grant 111-2-14. The work of Zheng Yang was also supported by the National Natural Science Foundation of China (No. 61571128). The work of Z. Ding was supported by the UK EPSRC under grant number EP/L025272/1 and by H2020-MSCA-RISE-2015 under grant number 690750. The work of N. Al-Dhahir was made possible by NPRP grant \# NPRP 8-627-2-260 from the Qatar National Research Fund. in order to remove the co-channel interference due to the implementation of NOMA [4] and [5].

The impact of partial channel state information on the performance of NOMA has been studied in [6], by assuming that users are uniformly distributed in a disk. The performance of NOMA in large-scale underlay cognitive radio (CR) networks with randomly deployed users was studied in [7]. The impact of user pairing on NOMA was investigated in [8], where two types of power allocation schemes were proposed. One is termed NOMA based on fixed power allocation (F-NOMA), and the other is termed CR inspired NOMA (CR-NOMA). The power allocation problem for realizing user fairness in NOMA systems has been investigated in [9], and the work in [10] considered the power allocation problem for NOMA with practical modulation based on finite constellation sizes. In [11], an amplify-and-forward (AF) relay equipped with a single antenna was used to help the base station for serving users equipped with multiple antennas in cooperative NOMA networks.

Simultaneous wireless information and power transfer (SWIPT) is a new paradigm, which can prolong the lifetime of energy constrained networks. Therefore, SWIPT is an important enabling technology to $5 \mathrm{G}$ communications, in order to support its Internet of Things (IoT) functionality. The key idea of SWIPT is to encourage nodes to harvest energy from their received radio frequency information-bearing signals, which was firstly proposed in [12]. However, it is not practical for a receiver to decode information and harvest energy at the same time, due to the practical limitation. Motivated by this issue, the authors in [13] have proposed two practical receiver architectures, i.e., power splitting (PS) and time switching (TS). The PS and TS protocol have been applied to cooperative networks with AF relaying, and their outage probability and throughputs have been analysed in [14]. In [15] and [16], game theoretic approaches for PS have been proposed in a multi-user cooperative decode-and-forward (DF) and AF relaying network, respectively. In [17], the author has studied the outage performance and the average harvested energy for SWIPT in large-scale cooperative networks. The work in [18][20] investigates the outage probability for the multiple relays with energy storage devices, where the Markov chain model is used to describe the states of the batteries.

Since NOMA improves the spectral efficiency of cooperative networks, and SWIPT provides more incentives for user cooperation, it is natural to consider the combination of NOMA and SWIPT in cooperative networks. An example for such an combination can be found in [21], where users with strong channel conditions were used as energy harvesting 
relays to improve the reception reliability for those users with poor channel conditions. In this paper, a different cooperative DF relaying scenario is considered, where one source communicates with two users via a dedicated relay which harvests energy from the source, and the NOMA principle is used for cooperative transmission. The main contribution of this paper is three-fold, as listed in the following:

- We first consider the application of SWIPT to cooperative NOMA with fixed power allocation, where the outage probability and diversity gain are considered as criteria to analyse the system performance. The developed closedform analytical results show that SWIPT-F-NOMA can achieve better outage performance than SWIPT-OMA under the condition that the power allocation coefficients and the users' targeted rates are chosen correctly.

- With the application of the cognitive radio concept, we consider two types of CR-NOMA with different constraints as explained in the following. One is to meet a fixed quality of service (QoS) requirement, such as a targeted data rate, at the user with worse channel condition (CR-NOMA(F)), and the other is to meet a dynamic QoS requirement at the user with strong channel conditions (CR-NOMA(D)), i.e., this user will experience a larger rate in NOMA than that in OMA. Furthermore, the SWIPT concept is applied into the two CR-NOMA cooperative networks. The exact expressions for the outage probability and their high signal-to-noise ratio (SNR) asymptotic results are derived. The analytical results show that SWIPT-CR-NOMA(F) achieves better outage performance for the user with poor channel conditions, but results in a loss of the outage probability for the user with strong channel conditions, compared to those of SWIPT-OMA. With SWIPT-CR-NOMA(D), the user with the strong channel conditions experiences the same outage probability as the case with SWIPT-OMA, but the user with the poor channel conditions experiences better outage performance compared to the case with SWIPTOMA.

- Furthermore, the analytical results developed in this paper demonstrate that the use of SWIPT-F-NOMA, SWIPTCR-NOMA(F), or SWIPT-CR-NOMA(D) will not cause any diversity gain loss, compared to the case that the relay uses its own battery for relay transmission power, but these SWIPT-NOMA schemes will lead to a loss of the outage probability. In addition, SWIPT-CR-NOMA(F) achieves the best outage performance for the user with the poor channel conditions, but the worst outage probability for the user with the strong channel conditions, among the three SWIPT-NOMA schemes.

\section{System ModeL}

Consider a cooperative network with one source $(S)$, one energy harvesting relay $(R)$, and two users $\left(U_{1}, U_{2}\right)$. Assume that all the nodes are equipped with a single antenna, and there is no direct link between the source and the users, i.e., the source communicates with the users via the energy harvesting relay. All the wireless channels are assumed to

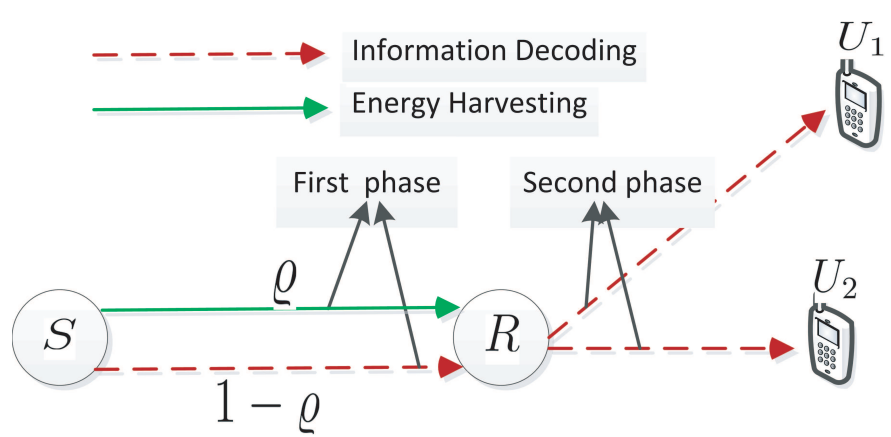

Fig. 1. System model of NOMA based cooperative network with SWIPT.

undergo quasi-static independent and non-identically Rayleigh fading, as shown in Fig. 1.

The cooperative transmission consists of two time slots. In the first time slot, the source $S$ uses superposition coding to combine two independent signals, $x_{1}$ and $x_{2}$, as $x=$ $\sqrt{\gamma_{1} P} x_{1}+\sqrt{\gamma_{2} P} x_{2}$, where $x_{k}, k=1,2$, is message for $U_{k}, P$ is the transmission power at source $S$, and $\gamma_{k}$ is the power allocation factor for $x_{k}$, with the constraint $\gamma_{1}+\gamma_{2}=1$. Therefore, during the first time slot, the receive signal at relay $R$ is given by

$$
y_{R}=\sqrt{(1-\varrho)}\left(\sqrt{\gamma_{1} P} x_{1}+\sqrt{\gamma_{2} P} x_{2}\right) h+w,
$$

where $\varrho$ is the power splitting coefficient, $w \sim \mathcal{C N}\left(0, \sigma^{2}\right)$ is the additive white Gaussian noise (AWGN), $h=g d^{-\frac{\alpha}{2}}$ is the channel coefficient between $S$ and $R, g$ denotes Rayleigh fading from $S$ to $R$ with $g \sim \mathcal{C N}(0,1), d$ is the distance between $S$ and $R$, and $\alpha$ is the path loss exponent.

Without loss of generality, assume that the relay detects the signal, $x_{1}$, first. Therefore, the achievable rates for the relay to detect signal $x_{1}$ is given by

$$
R_{1}=\frac{1}{2} \log _{2}\left(1+\frac{(1-\varrho) \gamma_{1} \rho|h|^{2}}{(1-\varrho) \gamma_{2} \rho|h|^{2}+1}\right),
$$

where $\rho=\frac{P}{\sigma^{2}}$ denotes the transmit SNR.

If the relay can successfully decode the message $x_{1}$, i.e., $R_{1} \geq R_{1}^{*}$, then it can remove the signal $x_{1}$ before detecting $x_{2}$, where $R_{1}^{*}$ denotes the targeted rate for $U_{1}$. This means that under the condition $R_{1} \geq R_{1}^{*}$, SIC is successfully carried out at the relay, and the message $x_{2}$ will be detected at the relay with the following instantaneous rate

$$
R_{2}=\frac{1}{2} \log _{2}\left(1+(1-\varrho) \gamma_{2} \rho|h|^{2}\right)
$$

It is noted that the power splitting protocol is applied at the relay to harvest energy from the source. In addition, the relay uses the DF protocol to decode the signals from the source. Because of these reasons, the relay has to firstly ensure the detection of the messages from the source, then it can carry out energy harvesting. In other words, only when the relay can successfully decode the signals from the source, it can use the harvested energy to forward the signals to the users. Therefore, it is much more important for the relay to decode the information than performing energy harvesting. 
In order to ensure that the relay can harvest the sufficient energy to power the relay transmission, we impose the following constrains, $R_{1}=R_{1}^{*}$ and $R_{2}=R_{2}^{*}$, which imply that relay $R$ can correctly decode both the signals $x_{1}$ and $x_{2}$, simultaneously, where $R_{2}^{*}$ denote the targeted data rates for $U_{2}$. With these imposed conditions, $R_{1}=R_{1}^{*}$ and $R_{2}=R_{2}^{*}$, i.e.,

$$
\left\{\begin{array}{l}
\frac{1}{2} \log _{2}\left(1+\frac{(1-\varrho) \gamma_{1} \rho|h|^{2}}{(1-\varrho) \gamma_{2} \rho|h|^{2}+1}\right)=R_{1}^{*} \\
\frac{1}{2} \log _{2}\left(1+(1-\varrho) \gamma_{2} \rho|h|^{2}\right)=R_{2}^{*}
\end{array}\right.
$$

After some algebraic manipulations, (4) can be rewritten as follows:

$$
\left\{\begin{array}{l}
(1-\varrho) \rho|h|^{2}\left(1-2^{2 R_{1}^{*}} \gamma_{2}\right)=2^{2 R_{1}^{*}}-1 \\
(1-\varrho) \rho|h|^{2} \gamma_{2}=2^{2 R_{2}^{*}}-1
\end{array}\right.
$$

According to (5), it is easy to obtain the power allocation factor $\gamma_{2}$ and the power splitting factor $\varrho$ which are given by

$$
\gamma_{2}=\frac{2^{2 R_{2}^{*}}-1}{2^{2 R_{1}^{*}+2 R_{2}^{*}}-1}
$$

and

$$
\varrho=\max \left\{0,1-\frac{2^{2 R_{1}^{*}+2 R_{2}^{*}}-1}{\rho|h|^{2}}\right\},
$$

respectively, note that $0<\gamma_{2}<1$, and $\varrho<1$. Hence $\varrho=0$ means the received signals from the source are fully used for information decoding, and the relay cannot harvest any energy.

Based on (1), the energy harvested at relay $R$ is given by [14], [15]

$$
P_{R}=\eta \varrho P|h|^{2},
$$

where $\eta$ is the energy harvesting coefficient. Note that the energy harvested from AWGN has been omitted.

Based on (6) and (7), the maximal transmission power at relay $R$ can be obtained as follows:

$$
P_{R}=\max \left\{0, \eta\left(P|h|^{2}-\varepsilon \sigma^{2}\right)\right\},
$$

where $\varepsilon=2^{2 R_{1}^{*}+2 R_{2}^{*}}-1$.

The reason for the simplified energy harvesting model in (9) is given as follows. On one hand, the contribution of this paper is to show that the use of NOMA can yield some significant outage performance gains, compared to OMA, when both of them use the same SWIPT protocol. On the other hand, the simplified SWIPT model can provide some insights to understand the advantage of combining SWIPT with NOMA, which can offer some technical guidances to implement SWIPT in practice. It is worth pointing out that it is a promising future direction to study the impact of the energy for maintaining the user circuits on the performance of SWIPT, which is beyond the scope of this paper.

Since both $x_{1}$ and $x_{2}$ are decoded correctly at the relay, the NOMA scheme can be used to serve the two users in the second time slot. Firstly, denote $h_{k}=g_{k} d_{k}^{-\frac{\alpha}{2}}, k=1,2$, is the channel coefficient between the relay and $U_{k}$, where $g_{k} \sim$ $\mathcal{C N}(0,1)$ and $d_{k}$ are Rayleigh fading and the distance from the relay to $U_{k}$, respectively. Without loss of generality, assume that the channel fading gains $\left|h_{k}\right|^{2}$ are ordered as $\left|h_{1}\right|^{2} \leq$ $\left|h_{2}\right|^{2}$. According to NOMA scheme, the relay retransmits a superimposed mixture of $x_{1}$ and $x_{2}$ as $x_{R}=\sqrt{\alpha_{1} P_{R}} x_{1}+$ $\sqrt{\alpha_{2} P_{R}} x_{2}$, where $\alpha_{k}, k=1,2$, is power allocation factor for $U_{k}$, with $\alpha_{1}>\alpha_{2}$ and $\alpha_{1}+\alpha_{2}=1$. The received signal at the $k$-th user, $k=1,2$, is given by

$$
y_{k}=\left(\sqrt{\alpha_{1} P_{R}} x_{1}+\sqrt{\alpha_{2} P_{R}} x_{2}\right) h_{k}+w_{k},
$$

where $w_{k}$ is AWGN with mean zero and variance $\sigma^{2}$.

Based on the NOMA principle, both users will decode $x_{1}$ first, since $\left|h_{1}\right|^{2} \leq\left|h_{2}\right|^{2}$. Therefore, the achievable rate for $U_{1}$ to detect $x_{1}$ is given by

$$
R_{1}^{N}=\frac{1}{2} \log _{2}\left(1+\frac{\alpha_{1} \rho_{R}\left|h_{1}\right|^{2}}{\alpha_{2} \rho_{R}\left|h_{1}\right|^{2}+1}\right),
$$

where $\rho_{R}=\frac{P_{R}}{\sigma^{2}}$.

The achievable rate for $U_{2}$ to detect $x_{1}$ is given by

$$
R_{1 \rightarrow 2}^{N}=\frac{1}{2} \log _{2}\left(1+\frac{\alpha_{1} \rho_{R}\left|h_{2}\right|^{2}}{\alpha_{2} \rho_{R}\left|h_{2}\right|^{2}+1}\right) .
$$

Note that if $R_{1 \rightarrow 2}^{N} \geq R_{1}^{*}$, i.e., $U_{2}$ can correctly decode the signal, $x_{1}$, which means that under the condition $R_{1 \rightarrow 2}^{N} \geq R_{1}^{*}$, the SIC can be successfully carried out at $U_{2}$ to remove the signal, $x_{1}$. Therefore, the achievable rate for $U_{2}$ to decode $x_{2}$ is given by

$$
R_{2}^{N}=\frac{1}{2} \log _{2}\left(1+\alpha_{2} \rho_{R}\left|h_{2}\right|^{2}\right) .
$$

We will investigate the outage performance of SWIPTNOMA with two types of power allocation schemes in the following two sections. The motivation for considering the outage as the performance evaluation criterion is explained in the following.

Recall that the bit error ratio (BER) is an important performance criterion which can be used to evaluate the errors that occur in a transmission system. However, it is difficult to obtain the BER in closed-form expressions, since it depends on which coding and modulation schemes are used. Fortunately, the outage probability can be evaluated in a simpler way and provides a tight bound on the BER, when optimal coding with an infinite length is used [22].

Furthermore, the outage probability is defined as the event that when the instantaneous rate at the receiver falls below a targeted date rate, which can be used to measure a predefined QoS requirement for delay-sensitive networks. This is because the throughput is a function of the channel fading quality, and evaluating the satisfied QoS requirement is quite challenging. Therefore, it is important to investigate the outage performance in wireless communication system, especially in delay-sensitive networks.

\section{NOMA BASED ON FIXED POWER AllocATION}

With F-NOMA, the power allocation factors for the users are constants and the strong user is allocated less power than the weak user, as long as the order of the users' channels is still the same. In this case, the power allocation coefficients are not functions of the users' channel fading gains, i.e., the transmitter only needs to know the order of the users' channel fading gains to perform NOMA. 
Recall that the relay harvests energy from the source and the SIC is carried out at $U_{2}$ to remove the signal $x_{1}$, before detecting its own message. Therefore the coverage probability for $U_{2}$ can be defined as follows:

$$
\overline{\mathrm{P}}_{2}^{F}=\operatorname{Pr}\left\{R_{1 \rightarrow 2}^{N} \geq R_{1}^{*}, R_{2}^{N} \geq R_{2}^{*}, P_{R}>0\right\} .
$$

The following theorem provides a closed-form expression for the outage probability experienced by $U_{2}$ and its approximation in the high SNR region, when F-NOMA relaying is used.

Theorem 1: The use of F-NOMA SWIPT relaying ensures that the following outage probability is achieved at $U_{2}$

$$
\mathrm{P}_{2}^{F}=1-e^{-\frac{d^{\alpha} \varepsilon}{\rho}}\left(\phi_{1} \mathrm{~K}_{1}\left(\phi_{1}\right)-\phi_{2} \mathrm{~K}_{1}\left(\phi_{2}\right)+\phi_{3} \mathrm{~K}_{1}\left(\phi_{3}\right)\right),
$$

where $\Psi_{\max }=\max \left\{\frac{\varepsilon_{1}}{\eta\left(\alpha_{1}-\alpha_{2} \varepsilon_{1}\right)}, \frac{\varepsilon_{2}}{\eta \alpha_{2}}\right\}, \alpha_{1}>\alpha_{2} \varepsilon_{1}, \varepsilon_{1}=$ $2^{2 R_{1}^{*}}-1, \varepsilon_{2}=2^{2 R_{2}^{*}}-1, \phi_{1}=\sqrt{\frac{4 \Psi_{\max } d^{\alpha} d_{1}^{\alpha}}{\rho}}, \phi_{2}=$ $\sqrt{\frac{4 \Psi_{\max } d^{\alpha}\left(d_{1}^{\alpha}+d_{2}^{\alpha}\right)}{\rho}}, \phi_{3}=\sqrt{\frac{4 \Psi_{\max } d^{\alpha} d_{2}^{\alpha}}{\rho}}$, and $\mathrm{K}_{v}()$ denotes the modified Bessel function of the second kind with order $v$ [23]. The high SNR approximation for $\mathrm{P}_{2}^{F}$ is given by

$$
\mathrm{P}_{2}^{F} \approx \frac{d^{\alpha}}{\rho}\left(\varepsilon-\Psi_{\max } d_{1}^{\alpha} \ln \frac{d_{1}^{\alpha}}{d_{1}^{\alpha}+d_{2}^{\alpha}}-\Psi_{\max } d_{2}^{\alpha} \ln \frac{d_{2}^{\alpha}}{d_{1}^{\alpha}+d_{2}^{\alpha}}\right) .
$$

\section{Proof: See Appendix A.}

Due to the fact that line-of-sight path of the S-R link may exist, we use Nakagami-m fadding for the S-R link to which SWIPT is applied, and investigate the outage performance for $U_{2}$ in SWIPT-F-NOMA systems. Particularly, assume that the S-R link, denoted by $h$, follows a Nakagami-m distribution with a sharp parameter $m$ and a variance $\lambda$, whereas the links from the relay to the users follow Rayleigh fading.

Most results in this paper can be extended to the case with Nakagami-m fading. For example, the outage probability for $U_{2}$ in SWIPT-F-NOMA is given in the following Lemma.

Lemma 1: The closed-form expression for the outage probability at $U_{2}$ in SWIPT-F-NOMA is given by

$$
\begin{aligned}
\widetilde{\mathrm{P}}_{2}^{F} & =1-\frac{e^{-\frac{m \varepsilon}{\lambda \rho}}}{\Gamma(m)} \sum_{i=0}^{m-1} 2^{-i}\left(\begin{array}{c}
m-1 \\
i
\end{array}\right)\left(\frac{m \varepsilon}{\lambda \rho}\right)^{m-1-i} \\
& \times\left(\Phi_{1}^{i+1} \mathrm{~K}_{i+1}\left(\Phi_{1}\right)-\Phi_{2}^{i+1} \mathrm{~K}_{i+1}\left(\Phi_{2}\right)+\Phi_{3}^{i+1} \mathrm{~K}_{i+1}\left(\Phi_{3}\right)\right),
\end{aligned}
$$

where $m$ is an integer, $\Phi_{1}=\sqrt{\frac{4 m \Psi_{\max } d_{1}^{\alpha}}{\lambda \rho}}, \Phi_{2}=$ $\sqrt{\frac{4 m \Psi_{\max }\left(d_{1}^{\alpha}+d_{2}^{\alpha}\right)}{\lambda \rho}}, \Phi_{3}=\sqrt{\frac{4 m \Psi_{\max } d_{2}^{\alpha}}{\lambda \rho}}$.

Proof: By using steps similar to those used to prove Theorem 1, and also using the fact that the PDF of $|h|^{2}$ is given by

$$
f_{|h|^{2}}(x)=\frac{m^{m}}{\Gamma(m) \lambda^{m}} x^{m-1} e^{-\frac{m x}{\lambda}},
$$

the Lemma is proved.

It can be observed from (17) that the closed-form expression can be found under the condition that $m$ is an integer, and it is difficult if $m$ is not an integer. It is important to point out that Rayleigh fading is a special case of Nakagami-m fading with $m=1$, as such, we adopt Rayleigh fading to model the link with SWIPT in the remaining of this paper, in order to evaluate the outage performance with mathematical tractability.

Similar to (14), the coverage probability at $U_{1}$ in F-NOMA SWIPT relaying transmission is defined as follows:

$$
\overline{\mathrm{P}}_{1}^{F}=\operatorname{Pr}\left\{R_{1}^{N} \geq R_{1}^{*}, P_{R}>0\right\} .
$$

Recall that the cumulative density function (CDF) of $\left|h_{1}\right|^{2}$ is given by

$$
F_{\left|h_{1}\right|^{2}}(x)=1-e^{-\left(d_{1}^{\alpha}+d_{2}^{\alpha}\right) x} .
$$

By using this $\mathrm{CDF}$ and also following the steps similar to those in the proof of Theorem 1, a closed-form expression for the outage probability experienced by $U_{1}$ in F-NOMA SWIPT relaying transmission and its high SNR approximation can be obtained as follows:

$$
\begin{aligned}
\mathrm{P}_{1}^{F} & =1-e^{-\frac{d^{\alpha} \varepsilon}{\rho}} \phi_{4} \mathrm{~K}_{1}\left(\phi_{4}\right) \\
& \approx \frac{\Psi_{1} d^{\alpha}\left(d_{1}^{\alpha}+d_{2}^{\alpha}\right)}{\rho} \ln \frac{\rho}{\Psi_{1} d^{\alpha}\left(d_{1}^{\alpha}+d_{2}^{\alpha}\right)},
\end{aligned}
$$

where $\Psi_{1}=\frac{\varepsilon_{1}}{\eta\left(\alpha_{1}-\alpha_{2} \varepsilon_{1}\right)}, \quad \alpha_{1}>\alpha_{2} \varepsilon_{1}$, and $\phi_{4}=$ $\sqrt{\frac{4 \Psi_{1} d^{\alpha}\left(d_{1}^{\alpha}+d_{2}^{\alpha}\right)}{\rho}}$.

The outage probability of OMA: OMA is a good benchmarking scheme for the NOMA transmission protocol. In cooperative SWIPT networks, if source communicates with $U_{i}, i=1,2$, via an energy harvesting relay in an OMA mode, such as time division multiple access (TDMA), four time slots are needed in total to serve $U_{1}$ and $U_{2}$. Similar to (9), the maximal transmission power for the $i$-th user is given by

$$
P_{R, i}=\max \left\{0, \eta\left(P|h|^{2}-\varepsilon_{i}^{\prime} \sigma^{2}\right)\right\},
$$

where $\varepsilon_{i}^{\prime}=2^{4 R_{i}^{*}}-1, i=1,2$.

The achievable rate for the energy harvesting relay communicating with $U_{i}$ in OMA is given by

$$
R_{i}^{T}=\frac{1}{4} \log _{2}\left(1+\rho_{R, i}\left|h_{i}\right|^{2}\right)
$$

where $\rho_{R, i}=\frac{P_{R, i}}{\sigma^{2}}$.

The use of the OMA strategy means that the outage probability at $U_{i}$ can be defined as follows:

$$
\begin{aligned}
\mathrm{P}_{i}^{O} & =1-\operatorname{Pr}\left\{\frac{1}{4} \log _{2}\left(1+\rho_{R, i}\left|h_{i}\right|^{2}\right)>R_{i}^{*}, P|h|^{2}>\varepsilon_{i}^{\prime} \sigma^{2}\right\} \\
& =1-\operatorname{Pr}\left\{\left|h_{i}\right|^{2}>\frac{\varepsilon_{i}^{\prime}}{\eta\left(\rho|h|^{2}-\varepsilon_{i}^{\prime}\right)},|h|^{2}>\frac{\varepsilon_{i}^{\prime}}{\rho}\right\}
\end{aligned}
$$

Based on (24) and following the steps similar to those in the proof for Theorem 1, the outage probability of $U_{1}$ in OMA and its high SNR approximation are given by

$$
\begin{aligned}
\mathrm{P}_{1}^{O} & =1-e^{-\frac{d^{\alpha} \varepsilon_{1}^{\prime}}{\rho}} \phi_{5} \mathrm{~K}_{1}\left(\phi_{5}\right) \\
& \approx \frac{\varepsilon_{1}^{\prime} d^{\alpha}\left(d_{1}^{\alpha}+d_{2}^{\alpha}\right)}{\eta \rho} \ln \frac{\eta \rho}{\varepsilon_{1}^{\prime} d^{\alpha}\left(d_{1}^{\alpha}+d_{2}^{\alpha}\right)},
\end{aligned}
$$

where $\phi_{5}=\sqrt{\frac{4 \varepsilon_{1}^{\prime} d^{\alpha}\left(d_{1}^{\alpha}+d_{2}^{\alpha}\right)}{\eta \rho}}$ 
Similarly, the outage probability of $U_{2}$ in OMA and its high SNR approximation are given by

$$
\begin{aligned}
\mathrm{P}_{2}^{O} & =1-e^{-\frac{d^{\alpha} \varepsilon_{2}^{\prime}}{\rho}}\left(\phi_{6} \mathrm{~K}_{1}\left(\phi_{6}\right)-\phi_{7} \mathrm{~K}_{1}\left(\phi_{7}\right)+\phi_{8} \mathrm{~K}_{1}\left(\phi_{8}\right)\right) \\
& \approx \frac{d^{\alpha}}{\rho}\left(\varepsilon-\varepsilon_{2}^{\prime} d_{1}^{\alpha} \ln \frac{d_{1}^{\alpha}}{d_{1}^{\alpha}+d_{2}^{\alpha}}-\varepsilon_{2}^{\prime} d_{2}^{\alpha} \ln \frac{d_{2}^{\alpha}}{d_{1}^{\alpha}+d_{2}^{\alpha}}\right),
\end{aligned}
$$

where $\phi_{6}=\sqrt{\frac{4 \varepsilon_{2}^{\prime} d^{\alpha} d_{1}^{\alpha}}{\eta \rho}}, \phi_{7}=\sqrt{\frac{4 \varepsilon_{2}^{\prime} d^{\alpha}\left(d_{1}^{\alpha}+d_{2}^{\alpha}\right)}{\eta \rho}}$, and $\phi_{8}=$ $\sqrt{\frac{4 \varepsilon_{2}^{\prime} d^{\alpha} d_{2}^{\alpha}}{\eta \rho}}$

One can observe from (16) and (26), (21) and (25) that FNOMA based SWIPT can achieve the same diversity gain as OMA based SWIPT. However, in Section V, both simulations and analytical results demonstrate that F-NOMA based SWIPT can realize a better outage performance compared to the OMA scheme. This is because the use of NOMA allows all the users to share the bandwidth resources, which yields higher spectral efficiency, compared to OMA.

\section{Cognitive Radio Inspired NOMA}

In this Section, we focus on two types of CR inspired NOMA. The first one is to treat the user with poor connections to the relay as a primary user, and the other one is to treat the user with strong connections to the relay as a primary user.

A. CR-NOMA with a fixed QoS requirement at the weak user

Theorem 1 shows that SWIPT-F-NOMA can achieve better performance than SWIPT-OMA under the condition $\alpha_{1}>$ $\alpha_{2} \varepsilon_{1}$, i.e., the power allocation factors and the users' targeted rates need to be carefully chosen. In order to overcome this disadvantage, we can consider NOMA as a special case of CR systems [8], where a user with poor channel conditions is considered as a primary user sharing the same spectrum with a secondary user, which has strong channel conditions. The advantage of this CR-NOMA is that the QoS requirement of the weak user can be strictly guaranteed.

Recall that the channel fading gains have been ordered as $\left|h_{1}\right|^{2} \leq\left|h_{2}\right|^{2}$, which means $U_{1}$ can be considered as a primary user and should be served with high priority based on the concept of CR networks [24]. Therefore, the rate at $U_{1}, R_{1}^{N}$, in (11) needs to satisfy the following constraint:

$$
\frac{1}{2} \log _{2}\left(1+\frac{\alpha_{1} \rho_{R}\left|h_{1}\right|^{2}}{\alpha_{2} \rho_{R}\left|h_{1}\right|^{2}+1}\right) \geq R_{1}^{*},
$$

which means that the maximal transmit power factor $\alpha_{2}$ can be used for $U_{2}$ is given by

$$
\alpha_{2}=\max \left\{0, \frac{\rho_{R}\left|h_{1}\right|^{2}-\varepsilon_{1}}{\rho_{R}\left|h_{1}\right|^{2}\left(1+\varepsilon_{1}\right)}\right\} .
$$

Based on the relay transmission power $P_{R}$ in (9) and the power allocation factor $\alpha_{2}$ in (28), a closed-form expression for the outage probability at $U_{1}$ in CR-NOMA can be obtained as follows:

$$
\begin{aligned}
\mathrm{P}_{1}^{C, I} & =1-\operatorname{Pr}\left\{|h|^{2}>\frac{\varepsilon}{\rho},\left|h_{1}\right|^{2}>\frac{\varepsilon_{1}}{\eta\left(\rho|h|^{2}-\varepsilon\right)}\right\} \\
& =1-\int_{\frac{\varepsilon}{\rho}}^{\infty} e^{-\frac{\left(d_{1}^{\alpha}+d_{2}^{\alpha}\right) \varepsilon_{1}}{\eta(\rho x-\varepsilon)}} d^{\alpha} e^{-d^{\alpha} x} d x \\
& =1-e^{-\frac{d^{\alpha} \varepsilon}{\rho}} \phi_{9} \mathrm{~K}_{1}\left(\phi_{9}\right),
\end{aligned}
$$

where $\phi_{9}=\sqrt{\frac{4 \varepsilon_{1} d^{\alpha}\left(d_{1}^{\alpha}+d_{2}^{\alpha}\right)}{\eta \rho}}$.

At high SNR, $\mathrm{P}_{1}^{C, I}$ can be approximated as follows:

$$
\mathrm{P}_{1}^{C, I} \approx \frac{\varepsilon_{1} d^{\alpha}\left(d_{1}^{\alpha}+d_{2}^{\alpha}\right)}{\eta \rho} \ln \frac{\eta \rho}{\varepsilon_{1} d^{\alpha}\left(d_{1}^{\alpha}+d_{2}^{\alpha}\right)} .
$$

Based on (9), (13), and (28), the coverage probability of $U_{2}$ in CR-NOMA $(F)$ relaying SWIPT transmission is defined as follows:

$$
\overline{\mathrm{P}}_{2}^{C, I}=\left\{R_{2}^{N} \geq R_{2}^{*}, \rho_{R}>0,\left|h_{1}\right|^{2}>\frac{\varepsilon_{1}}{\rho_{R}}\right\} .
$$

The following theorem provides an exact expression for the outage probability experienced by $U_{2}$ and its approximation at high SNR.

Theorem 2: The use of CR-NOMA with a fixed QoS constraint at $U_{1}$ yields an outage probability at $U_{2}$ as follows:

$$
\begin{aligned}
\mathrm{P}_{2}^{C, I}=1 & -e^{-\frac{d^{\alpha} \varepsilon}{\rho}} \sqrt{\frac{\tau}{\rho}} \mathrm{K}_{1}\left(\sqrt{\frac{\tau}{\rho}}\right)-\sum_{i_{1}, i_{2} \in\{1,2\}} \frac{2 d^{\alpha} d_{i_{1}}^{\alpha} e^{-\frac{d^{\alpha} \varepsilon}{\rho}}}{\eta \rho} \\
& \times \int_{0}^{\varepsilon_{2}\left(1+\varepsilon_{1}\right)} \mathrm{K}_{0}\left(2 \sqrt{\frac{g_{i_{1}, i_{2}}(s)}{\rho}}\right) d s
\end{aligned}
$$

where $g_{i_{1}, i_{2}}(s)=\frac{d^{\alpha} d_{i_{2}}^{\alpha} \varepsilon_{2}\left(1+\varepsilon_{1}\right)\left(s+\varepsilon_{1}\right)+d^{\alpha} d_{i_{1}}^{\alpha} s\left(s+\varepsilon_{1}\right)}{\eta s}, \tau=$ $\frac{4 d^{\alpha}\left(d_{1}^{\alpha}+d_{2}^{\alpha}\right)\left(\varepsilon_{1}+\varepsilon_{2}\left(1+\varepsilon_{1}\right)\right)}{\eta}$. When $\rho \rightarrow \infty$, the high SNR approximation of the outage probability is given by

$$
\mathrm{P}_{2}^{C, I} \approx \frac{\ln \rho}{\rho}
$$

Proof: See Appendix B.

Comparing (33) to (26), we can see that the diversity gain at $U_{2}$ in SWIPT-CR-NOMA(F) is identical to that in SWIPTOMA, but the use of CR-NOMA results in a higher outage probability, compares to the OMA case. The main reason is that $U_{2}$ is regarded as a secondary user in SWIPT-CR$\operatorname{NOMA}(\mathrm{F})$, and it is served only after the QoS requirement of $U_{1}$ is satisfied.

\section{B. CR-NOMA with a dynamic QoS requirement at the strong user}

Recall that by using this CR-NOMA inspired power allocation policy, the strong user can be served by the transmitter only when the weak user's QoS is met. Especially, when the weak user's targeted rate is very high, the transmitter has to allocate all the power to support this user, and the strong user cannot be served. In order to ensure that the strong user in NOMA systems can be also served, we impose a constraint that this user achieves at least the same throughput as that in OMA, then allocate all the remaining power to the weak user. In other words, the user with strong channel conditions is regarded as a primary user, and consider a dynamic QoS requirement of this user which is to achieve a rate larger than that of OMA, i.e.,

$$
\frac{1}{2} \log _{2}\left(1+\alpha_{2} \rho_{R}\left|h_{2}\right|^{2}\right) \geq \frac{1}{4} \log _{2}\left(1+\rho_{R, 2}\left|h_{2}\right|^{2}\right),
$$


which means the maximal power allocation factor $\alpha_{1}$ is given by

$$
\alpha_{1}=1-\frac{\sqrt{1+\rho_{R, 2}\left|h_{2}\right|^{2}}-1}{\rho_{R}\left|h_{2}\right|^{2}} .
$$

Based on (35), the coverage probability of $U_{2}$ in CR-NOMA with a dynamic QoS requirement at the strong user (SWIPTCR-NOMA(D)) can be expressed as follows:

$$
\begin{aligned}
& \overline{\mathrm{P}}_{2}^{C, I I}= \operatorname{Pr}\left\{\frac{1}{2} \log _{2}\left(1+\frac{\alpha_{1} \rho_{R}\left|h_{2}\right|^{2}}{\alpha_{2} \rho_{R}\left|h_{2}\right|^{2}+1}\right) \geq R_{1}^{*},\right. \\
&\left.\frac{1}{2} \log _{2}\left(1+\alpha_{2} \rho_{R}\left|h_{2}\right|^{2}\right) \geq R_{2}^{*}\right\} \\
&=\operatorname{Pr}\left\{\frac{1}{4} \log _{2}\left(1+\rho_{R, 2}\left|h_{2}\right|^{2}\right) \geq R_{1}^{*},\right. \\
&\left.\frac{1}{4} \log _{2}\left(1+\rho_{R, 2}\left|h_{2}\right|^{2}\right) \geq R_{2}^{*}\right\} \\
&=\operatorname{Pr}\left\{\frac{1}{4} \log _{2}\left(1+\rho_{R, 2}\left|h_{2}\right|^{2}\right) \geq R_{2}^{*}\right\} .
\end{aligned}
$$

From (36), it is interesting to observe that $U_{2}$ in SWIPT-CRNOMA(D) can experience the same coverage probability as that of SWIPT-OMA. The reason for this is that the maximal power allocation factor $\alpha_{1}$ in (35) is based on the condition in (34), i.e., $U_{2}$ should get a rate no less than that in OMA.

Theorem 3: The use of CR-NOMA with a dynamic QoS requirement at the strong user yields an outage probability at $U_{1}$ as expressed in (37) at the top of the nex$\mathrm{t}$ page, where $\rho_{R, 2}=\eta\left(\rho z-\varepsilon_{2}^{\prime}\right), \rho_{R}=\eta(\rho z-\varepsilon)$, $H(x)=\frac{\rho_{R, 2}-2 g(x)}{g^{2}(x)}, g(x)=\frac{\rho_{R} x-\varepsilon_{1}}{\left(\varepsilon_{1}+1\right) x}, K\left(\rho_{R, 2}, \rho_{R}\right)=$ $\frac{\rho_{R, 2}\left(\varepsilon_{1}+1\right)^{2}-2 \rho_{R}+\sqrt{\Delta\left(\rho_{R, 2}, \rho_{R}\right)}}{2 \rho_{R}^{2}}, \Delta\left(\rho_{R, 2}, \rho_{R}\right)=\left(\rho_{R, 2}\left(\varepsilon_{1}+\right.\right.$ $\left.1)^{2}-2 \rho_{R}\right)^{2}+4 \rho_{R}^{2}\left(\varepsilon_{1}^{2}+2 \varepsilon_{1}\right)$. At high SNR, $\mathrm{P}_{1}^{C, I I}$ can be approximated as follows:

$$
\mathrm{P}_{1}^{C, I I} \approx \frac{\ln \rho}{\rho} .
$$

Proof: See Appendix C.

Note that the SWIPT-CR-NOMA(D) achieves similar outage performance compared to SWIPT-OMA at high SNR, as the approximated expression in (38) is quite similar to that in (25) at high SNR. However, the simulation results in Section $\mathrm{V}$ show that the outage probability of $U_{1}$ in SWIPT-CRNOMA(D) is always better than that in SWIPT-OMA. The main reason is that the power allocation factor, $\alpha_{1}$, in (35) is a function of the strong user's channel gain, and the rate for $U_{1}, R_{1}^{N}$, in (11) is an increasing function of $\alpha_{1}$, which can further improve the weak user's performance.

\section{Numerical Results}

In this section, computer simulations are used to verify the accuracy of the developed analytical results about the outage probability of NOMA based cooperative SWIPT networks. In the considerable network, we choose the energy harvesting efficiency $\eta=0.4$, the path loss factor $\alpha=4$. The carrier frequency, transmitter antenna gain, and receiver antenna gain are set to be $915 \mathrm{MHz}, 1 \mathrm{dBm}$, and $1 \mathrm{dBm}$, respectively. Fig. 2-Fig. 4 are obtained with the following distances, where the distance between the source and the relay is given by $d=$

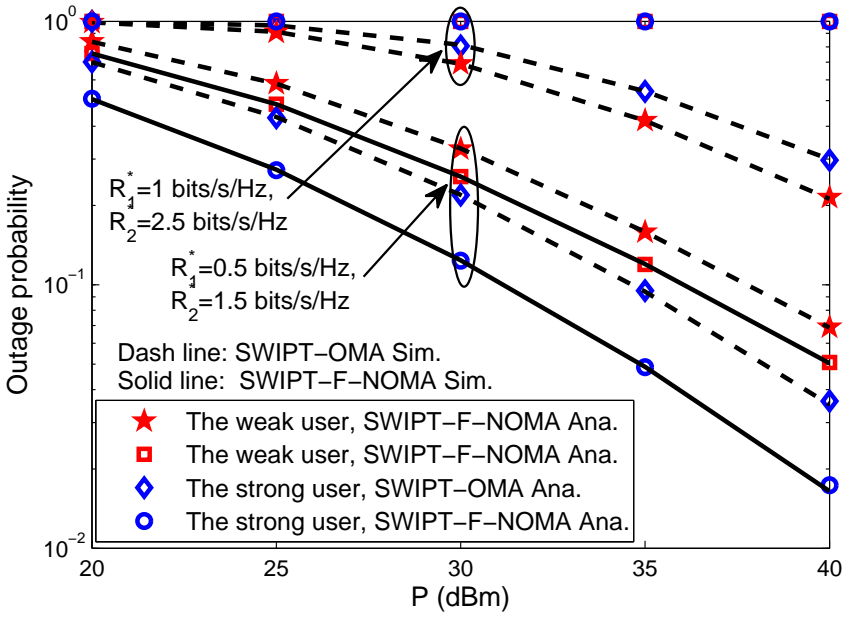

Fig. 2. SWIPT-F-NOMA with $\alpha_{1}=0.75$ and $\alpha_{2}=0.25$.

$10 \mathrm{~m}$, the distances from relay to $U_{1}$ and $U_{2}$ are given by $d_{1}=25 \mathrm{~m}$ and $d_{2}=10 \mathrm{~m}$, respectively, and the noise power is set to be $-114 \mathrm{dBm}$. Fig. 5 is obtained with the following distances, where the distance between the source and the relay is given by $d=5 \mathrm{~m}$, the distances from the relay to the $U_{1}$ and the $U_{2}$ are given by $d_{1}=16 \mathrm{~m}$ and $d_{2}=5 \mathrm{~m}$, respectively, and the noise power is set to be $-84 \mathrm{dBm}$.

Fig. 2 shows the outage performance of F-NOMA with SWIPT in cooperative networks as a function of the transmitter power $P$. It can be seen from Fig. 2 that SWIPT-F-NOMA achieves the same diversity gain as SWIPT-OMA, but the NOMA scheme offers a constant performance gain in the outage probability, compared to the OMA scheme. This is because NOMA allows multiple users to share the same resources at the time, frequency and code domains via power domain multiplexing. Furthermore, if the users' targeted rates and power allocation factors cannot satisfy $\alpha_{1}>\alpha_{2} \varepsilon_{1}$, the outage will always occur, as stated in Theorem 1. In addition, it is important to point out that the curves for the closed-form expressions in (15), (21), (25) and (26) match Monte Carlo simulation results perfectly.

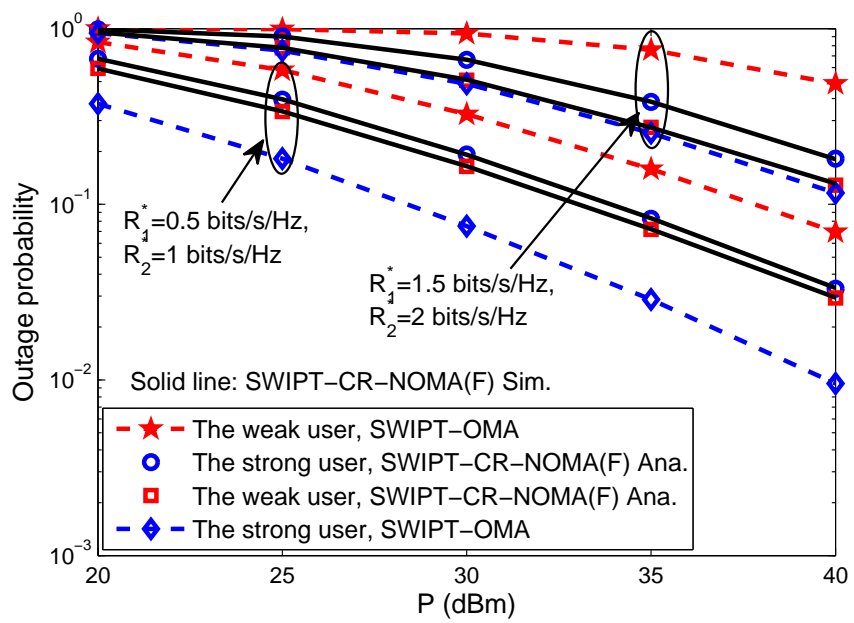

Fig. 3. SWIPT-CR-NOMA with a fixed QoS requirement at the weak user. 


$$
\begin{aligned}
\mathrm{P}_{1}^{C, I I} & =1-d^{\alpha} \int_{\frac{\varepsilon_{2}^{\prime}}{\rho}}^{\infty}\left(e^{-\left(d_{1}^{\alpha}+d_{2}^{\alpha}\right) K\left(\rho_{R, 2}, \rho_{R}\right)}-e^{-\frac{\left(d_{1}^{\alpha}+d_{2}^{\alpha}\right) \varepsilon_{1}}{\eta(\rho z-\varepsilon)}}\right) e^{-d^{\alpha} z} d z-e^{-\frac{d^{\alpha} \varepsilon}{\rho}} \sqrt{\frac{4 d^{\alpha}\left(d_{1}^{\alpha}+d_{2}^{\alpha}\right) \varepsilon_{1}}{\eta \rho}} \mathbf{K}_{1}\left(\sqrt{\frac{4 d^{\alpha}\left(d_{1}^{\alpha}+d_{2}^{\alpha}\right) \varepsilon_{1}}{\eta \rho}}\right) \\
& -\sum_{i_{1}, i_{2} \in\{1,2\}} d_{i_{1}}^{\alpha} \int_{\frac{\varepsilon_{2}^{\prime}}{\rho}}^{\infty} \int_{\frac{\varepsilon_{1}}{\rho_{R}}}^{K\left(\rho_{R, 2}, \rho_{R}\right)} e^{-d_{i_{2}}^{\alpha} H(x)-d_{i_{1}}^{\alpha} x} d x d^{\alpha} e^{-d^{\alpha} z} d z
\end{aligned}
$$

In Fig. 3, the outage probability of SWIPT-CR-NOMA with a fixed QoS constraint at the weak user is investigated. Fig. 3 demonstrates that all the curves are parallel, which means the achievable diversity order is the same for SWIPT-CR$\operatorname{NOMA}(\mathrm{F})$ and SWIPT-OMA. Compared to SWIPT-OMA, it is interesting to find that the weak user in SWIPT-CR-NOMA(F) can significantly improve its outage performance, while the strong user's outage probability is deteriorated severely. This is because the power allocation factor, $\alpha_{2}$ in (28) is a function of the weak user's channel gain, and the strong user cannot get sufficient power, especially when the weak user's targeted rate is high. Again, Fig. 3 confirms that the analytical results developed in (29) and (32) perfectly match computer simulations.

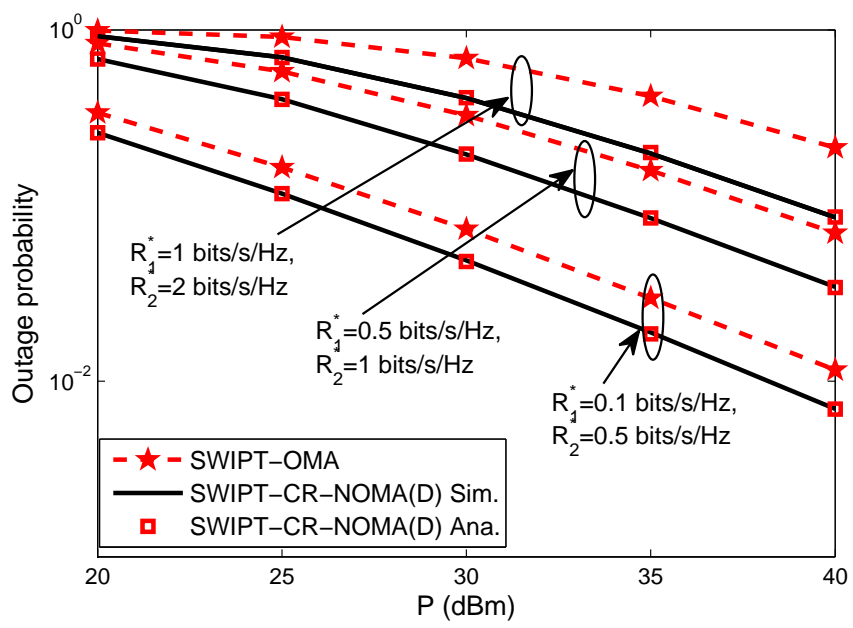

Fig. 4. The outage probability of weak user in SWIPT-CR-NOMA(D) with a dynamic QoS requirement at the strong user.

In Fig. 4, we focus on the outage performance of the user with weak channel conditions when the CR-NOMA power allocation policy is designed to meet the dynamic QoS constraint at the strong user. Note that there is no need to show the performance of $U_{2}$, since this strong user experiences the same outage performance as in SWIPT-OMA. As can be observed from Fig. 4, SWIPT-CR-NOMA(D) can achieve better outage performance than SWIPT-OMA, although the same diversity gain is realized by both schemes. Furthermore, Fig. 4 also demonstrates that the users' targeted rates significantly affect the performance gap between NOMA and OMA. Particularly, as the difference between $R_{1}^{*}$ and $R_{2}^{*}$ increases, the outage performance gap between SWIPT-CR-NOMA(D) and SWIPTOMA enlarges accordingly. In addition, computer simulations also confirm the accuracy of the analytical results developed in (37).

In Fig. 5, the outage probability difference between the

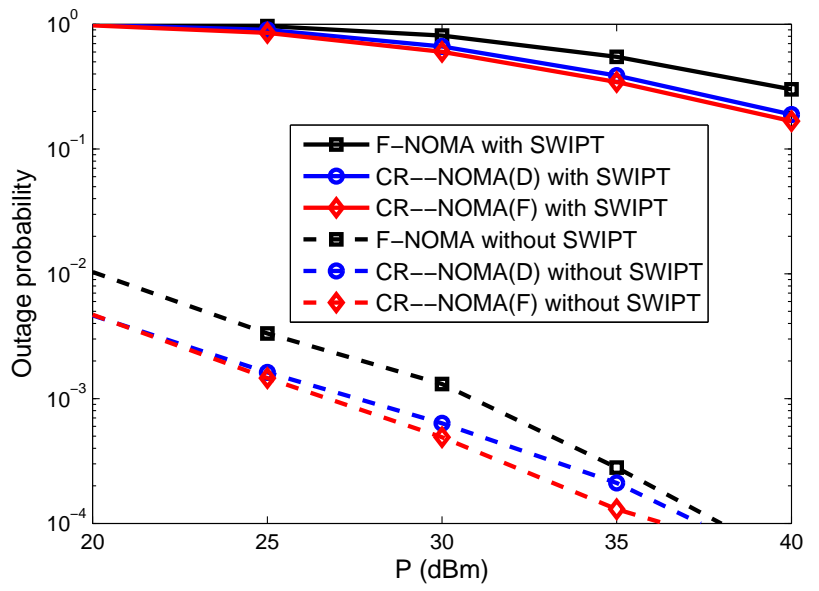

(a) The weak user.

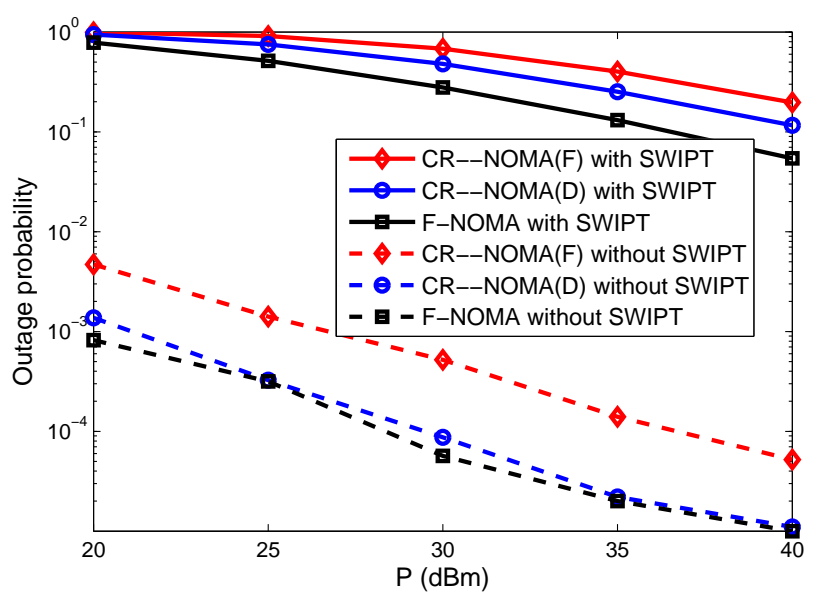

(b) The strong user.

Fig. 5. Outage probability comparision with/without SWIPT for CRNOMA and F-NOMA, where $\gamma_{1}=\alpha_{1}=0.7, \gamma_{2}=\alpha_{2}=0.3$, $R_{1}^{*}=0.5 \mathrm{bits} / \mathrm{s} / \mathrm{Hz}, R_{2}^{*}=1.5 \mathrm{bits} / \mathrm{s} / \mathrm{Hz}$.

NOMA schemes with SWIPT and without SWIPT is studied. Fig. 5 demonstrates that the diversity gain achieved by NOMA with SWIPT is the same as that achieved by NOMA without energy harvesting, but the use of SWIPT results in a outage probability loss. This phenomenon is expected since the use of SWIPT means that the relay transmission power which is harvested from the signals sent by the source is much smaller than that in the case without SWIPT. In addition, it can be seen from Fig. 5 that SWIPT-CR-NOMA(F) achieves the best outage performance for the weak user, but the worst outage probability for the strong user. 


\section{CONCLUSIONS}

We have investigated the application of SWIPT to cooperative F-NOMA and CR-NOMA networks, and their outage probabilities have been analyzed in this paper. Compared to SWIPT-OMA, SWIPT-F-NOMA can achieve better outage performance if the users' targeted rates and power allocation coefficients are correctly chosen. For SWIPT-CR-NOMA(F), the outage performance of the user with strong channel conditions is degraded compared to the OMA case, but the outage performance of the weak user can be significantly improved. Furthermore, for CR-NOMA-SWIPT(D), the weak user' outage performance improves evidently and there is no loss in the outage performance at the user with strong channel conditions, compared to SWIPT-OMA.

In this paper, the effect of power allocation on cooperative NOMA with SWIPT has been investigated, where all the nodes are equipped with a single antenna, and an important future direction is to consider the application of multiple-input multiple-output technologies to the addressed cooperative SWIPT-NOMA networks. In addition, another promising future direction is that the addressed system model in this paper can be extended to the case with multiple relays to improve the reception reliability of NOMA transmission. The key step is to consider how many relays can correctly decode the source signals and harvest energy from the source simultaneously, then choose one relay from the qualified relays to serve two users with different power allocation schemes.

\section{APPENDIX A}

\section{PROOF OF THEOREM 1}

Based on (14), the coverage probability of $U_{2}$ can be rewritten as follows:

$$
\begin{aligned}
\overline{\mathrm{P}}_{2}^{F}= & \operatorname{Pr}\left\{\frac{1}{2} \log _{2}\left(1+\frac{\alpha_{1} \rho_{R}\left|h_{2}\right|^{2}}{\alpha_{2} \rho_{R}\left|h_{2}\right|^{2}+1}\right) \geq R_{1}^{*},\right. \\
& \left.\frac{1}{2} \log _{2}\left(1+\alpha_{2} \rho_{R}\left|h_{2}\right|^{2}\right) \geq R_{2}^{*}, P_{R}>0\right\} \\
= & \operatorname{Pr}\left\{\left(\rho|h|^{2}-\varepsilon\right)\left|h_{2}\right|^{2}>\Psi_{\max },|h|^{2}>\frac{\varepsilon}{\rho}\right\} .
\end{aligned}
$$

Since the unordered channel fading gains $\left|\tilde{h}_{k}\right|^{2}, k=1,2$ are exponentially distributed with different expectations, the CDF of $\left|h_{2}\right|^{2}$ is given by

$$
F_{\left|h_{2}\right|^{2}}(y)=\left(1-e^{-d_{1}^{\alpha} y}\right)\left(1-e^{-d_{2}^{\alpha} y}\right) .
$$

Based on (40), the coverage probability $\overline{\mathrm{P}}_{2}^{F}$ in (39) can be calculated as follows:

$$
\begin{aligned}
\overline{\mathrm{P}}_{2}^{F}= & \int_{\frac{\varepsilon}{\rho}}^{\infty}\left(e^{-\frac{d_{1}^{\alpha} \Psi_{\text {max }}}{\rho x-\varepsilon}}+e^{-\frac{d_{2}^{\alpha} \Psi_{\text {max }}}{\rho x-\varepsilon}}-e^{-\frac{\left(d_{1}^{\alpha}+d_{2}^{\alpha}\right) \Psi_{\text {max }}}{\rho x-\varepsilon}}\right) \\
& \times d^{\alpha} e^{-d^{\alpha} x} d x .
\end{aligned}
$$

Let $t=\rho x-\varepsilon$, the above $\overline{\mathrm{P}}_{2}^{F}$ can be further evaluated as follows:

$$
\begin{aligned}
\overline{\mathrm{P}}_{2}^{F}= & \frac{e^{-\frac{d^{\alpha} \varepsilon}{\rho}}}{\rho} \int_{0}^{\infty}\left(e^{-\frac{d_{1}^{\alpha} \Psi_{\max }}{t}}+e^{-\frac{d_{2}^{\alpha} \Psi_{\max }}{t}}\right. \\
& \left.-e^{-\frac{\left(d_{1}^{\alpha}+d_{2}^{\alpha}\right) \Psi_{\max }}{t}}\right) d^{\alpha} e^{-\frac{d^{\alpha} t}{\rho}} d t \\
= & e^{-\frac{d^{\alpha} \varepsilon}{\rho}}\left[\sqrt{\frac{4 \Psi_{\max } d^{\alpha} d_{1}^{\alpha}}{\rho}} \mathrm{K}_{1}\left(\sqrt{\frac{4 \Psi_{\max } d^{\alpha} d_{1}^{\alpha}}{\rho}}\right)\right. \\
& -\sqrt{\frac{4 \Psi_{\max } d^{\alpha}\left(d_{1}^{\alpha}+d_{2}^{\alpha}\right)}{\rho}} \mathrm{K}_{1}\left(\sqrt{\frac{4 \Psi_{\max } d^{\alpha}\left(d_{1}^{\alpha}+d_{2}^{\alpha}\right)}{\rho}}\right) \\
+ & \left.\sqrt{\frac{4 \Psi_{\max } d^{\alpha} d_{2}^{\alpha}}{\rho}} \mathrm{K}_{1}\left(\sqrt{\frac{4 \Psi_{\max } d^{\alpha} d_{2}^{\alpha}}{\rho}}\right)\right]
\end{aligned}
$$

Substituting (42) into the outage probability $\mathrm{P}_{2}^{F}=1-\overline{\mathrm{P}}_{2}^{F}$, the first part of the theorem is proved.

When $z \rightarrow 0$, the Bessel function $z \mathrm{~K}_{1}(z)$ can be approximated by using series representation as follows [23, eq. (8.446)]:

$$
\begin{aligned}
z \mathrm{~K}_{1}(z)= & +\sum_{k=0}^{\infty} \frac{\left(\frac{z}{2}\right)^{2 k+1} z}{k ! \Gamma(k+2)}\left(\ln \frac{z}{2}+\mathbf{C}\right) \\
& -\frac{1}{2} \sum_{l=0}^{\infty} \frac{\left(\frac{z}{2}\right)^{2 l+1} z}{l !(n+l) !}\left(\sum_{k=1}^{l} \frac{1}{k}+\sum_{k=1}^{l+1} \frac{1}{k}\right) \\
\approx & 1+\frac{z^{2}}{2} \ln \frac{z}{2} .
\end{aligned}
$$

When $z \rightarrow 0$, the exponential function $e^{-z}$ can be approximated as follows:

$$
e^{-z} \approx 1-z
$$

By applying (43) and (44) into (15), $\mathrm{P}_{2}^{F}$ can be approximated at high SNR as follows:

$$
\begin{aligned}
\mathrm{P}_{2}^{F} \approx & 1-\left(1-\frac{\varepsilon d^{\alpha}}{\rho}\right)\left[\left(1+\frac{2 \Psi_{\max } d^{\alpha} d_{1}^{\alpha}}{\rho} \ln \sqrt{\frac{\Psi_{\max } d^{\alpha} d_{1}^{\alpha}}{\rho}}\right)\right. \\
& -\left(1+\frac{2 \Psi_{\max } d^{\alpha}\left(d_{1}^{\alpha}+d_{2}^{\alpha}\right)}{\rho} \ln \sqrt{\frac{\Psi_{\max } d^{\alpha}\left(d_{1}^{\alpha}+d_{2}^{\alpha}\right)}{\rho}}\right) \\
& \left.+\left(1+\frac{2 \Psi_{\max } d^{\alpha} d_{2}^{\alpha}}{\rho} \ln \sqrt{\frac{\Psi_{\max } d^{\alpha} d_{2}^{\alpha}}{\rho}}\right)\right] \\
& \approx \frac{d^{\alpha}}{\rho}\left(\varepsilon-\Psi_{\max } d_{1}^{\alpha} \ln \frac{d_{1}^{\alpha}}{d_{1}^{\alpha}+d_{2}^{\alpha}}-\Psi_{\max } d_{2}^{\alpha} \ln \frac{d_{2}^{\alpha}}{d_{1}^{\alpha}+d_{2}^{\alpha}}\right) .
\end{aligned}
$$

Therefore, the second part of the theorem is also proved. The proof for the theorem is complete. 


\section{APPENDIX B}

\section{PROOF OF THEOREM 2}

Based on (9) and (13), the coverage probability of $U_{2}$ in (31) can be further expressed as follows:

$$
\begin{aligned}
& \overline{\mathrm{P}}_{2}^{C, I}=\operatorname{Pr}\left\{\frac{1}{2} \log _{2}\left(1+\alpha_{2} \rho_{R}\left|h_{2}\right|^{2}\right) \geq R_{2}^{*}, \rho_{R}>0,\left|h_{1}\right|^{2}>\frac{\varepsilon_{1}}{\rho_{R}}\right\} \\
&=\operatorname{Pr}\left\{\frac{\eta\left(\rho|h|^{2}-\varepsilon\right)\left|h_{1}\right|^{2}-\varepsilon_{1}}{\left|h_{1}\right|^{2}\left(1+\varepsilon_{1}\right)}\left|h_{2}\right|^{2} \geq \varepsilon_{2},|h|^{2}>\frac{\varepsilon}{\rho}\right. \\
&\left.\left|h_{1}\right|^{2}>\frac{\varepsilon_{1}}{\eta\left(\rho|h|^{2}-\varepsilon\right)}\right\} .
\end{aligned}
$$

Recall that the users' channel gains are ordered as $\left|h_{1}\right|^{2} \leq$ $\left|h_{2}\right|^{2}$, the above coverage probability $\overline{\mathrm{P}}_{2}^{C, I}$ can be further rewritten as follows:

$$
\begin{aligned}
\overline{\mathrm{P}}_{2}^{C, I}= & \operatorname{Pr}\left\{\left|h_{2}\right|^{2} \geq\left|h_{1}\right|^{2},|h|^{2}>\frac{\varepsilon}{\rho},\left|h_{1}\right|^{2}>\frac{\varepsilon_{1}+\varepsilon_{2}\left(1+\varepsilon_{1}\right)}{\eta\left(\rho|h|^{2}-\varepsilon\right)}\right\} \\
+ & \operatorname{Pr}\left\{\left|h_{2}\right|^{2} \geq \frac{\varepsilon_{2}\left(1+\varepsilon_{1}\right)\left|h_{1}\right|^{2}}{\eta\left(\rho|h|^{2}-\varepsilon\right)\left|h_{1}\right|^{2}-\varepsilon_{1}},|h|^{2}>\frac{\varepsilon}{\rho}\right. \\
& \left.\frac{\varepsilon_{1}}{\eta\left(\rho|h|^{2}-\varepsilon\right)}<\left|h_{1}\right|^{2}<\frac{\varepsilon_{1}+\varepsilon_{2}\left(1+\varepsilon_{1}\right)}{\eta\left(\rho|h|^{2}-\varepsilon\right)}\right\} .
\end{aligned}
$$

Note that the joint probability density function (PDF) of $\left|h_{1}\right|^{2}$ and $\left|h_{2}\right|^{2}$ is given by [25]

$$
f_{\left|h_{1}\right|^{2},\left|h_{2}\right|^{2}}(x, y)=\sum_{i_{1}, i_{2} \in\{1,2\}} d_{i_{1}}^{\alpha} e^{-d_{i_{1}}^{\alpha} x} d_{i_{2}}^{\alpha} e^{-d_{i_{2}}^{\alpha} y},
$$

where $0<x<y$.

Denote the first factor in $\overline{\mathrm{P}}_{2}^{C R}$ in (47) as $Q_{1}$, which can be evaluated by applying (48) as follows:

$$
\begin{aligned}
Q_{1}= & \sum_{i_{1}, i_{2} \in\{1,2\}} d^{\alpha} d_{i_{1}}^{\alpha} d_{i_{2}}^{\alpha} \int_{\frac{\varepsilon}{\rho}}^{\infty} \int_{\frac{\varepsilon_{1}+\varepsilon_{2}\left(1+\varepsilon_{1}\right)}{\eta(\rho z-\varepsilon)}}^{\infty} \int_{x}^{\infty} e^{-d_{i_{2}}^{\alpha} y} d y \\
& \times e^{-d_{i_{1}}^{\alpha} x} d x e^{-d^{\alpha} z} d z \\
= & \sum_{i_{1}, i_{2} \in\{1,2\}} \frac{d^{\alpha} d_{i_{1}}^{\alpha}}{d_{i_{1}}^{\alpha}+d_{i_{2}}^{\alpha}} \int_{\frac{\varepsilon}{\rho}}^{\infty} e^{-\left(d_{i_{1}}^{\alpha}+d_{i_{2}}^{\alpha}\right) \frac{\varepsilon_{1}+\varepsilon_{2}\left(1+\varepsilon_{1}\right)}{\eta(\rho z-\varepsilon)}} e^{-d^{\alpha} z} d z \\
= & \sum_{i_{1}, i_{2} \in\{1,2\}} \frac{d_{i_{1}}^{\alpha} e^{-\frac{d^{\alpha} \varepsilon}{\rho}}}{d_{i_{1}}^{\alpha}+d_{i_{2}}^{\alpha}} \sqrt{\frac{4 d^{\alpha}\left(d_{i_{1}}^{\alpha}+d_{i_{2}}^{\alpha}\right)\left(\varepsilon_{1}+\varepsilon_{2}\left(1+\varepsilon_{1}\right)\right)}{\eta \rho}} \\
& \times \mathrm{K}_{1}\left(\sqrt{\frac{4 d^{\alpha}\left(d_{i_{1}}^{\alpha}+d_{i_{2}}^{\alpha}\right)\left(\varepsilon_{1}+\varepsilon_{2}\left(1+\varepsilon_{1}\right)\right)}{\eta \rho}}\right) \\
= & e^{-\frac{d^{\alpha} \varepsilon}{\rho}} \sqrt{\frac{4 d^{\alpha}\left(d_{1}^{\alpha}+d_{2}^{\alpha}\right)\left(\varepsilon_{1}+\varepsilon_{2}\left(1+\varepsilon_{1}\right)\right)}{\eta \rho}} \\
& \times K_{1}\left(\sqrt{\frac{4 d^{\alpha}\left(d_{1}^{\alpha}+d_{2}^{\alpha}\right)\left(\varepsilon_{1}+\varepsilon_{2}\left(1+\varepsilon_{1}\right)\right)}{\eta \rho}} .\right.
\end{aligned}
$$

Denote the second factor in $\overline{\mathrm{P}}_{2}^{C, I}$ in (47) as $Q_{2}$, which can be evaluated by applying (48) as follows:

$$
\begin{aligned}
Q_{2}= & \sum_{i_{1}, i_{2} \in\{1,2\}} \int_{\frac{\varepsilon}{\rho}}^{\infty} \int_{\frac{\varepsilon_{1}}{\eta(\rho z-\varepsilon)}}^{\frac{\varepsilon_{1}+\varepsilon_{2}\left(1+\varepsilon_{1}\right)}{\eta(\rho z-\varepsilon)}} \int_{\frac{\varepsilon_{2}\left(1+\varepsilon_{1}\right) x}{\eta(\rho z-\varepsilon) x-\varepsilon_{1}}}^{\infty} d_{i_{2}}^{\alpha} e^{-d_{i_{2}}^{\alpha} y} d y \\
& \times d_{i_{1}}^{\alpha} e^{-d_{i_{1}}^{\alpha} x} d x d^{\alpha} e^{-d^{\alpha} z} d z
\end{aligned}
$$

Let $t=\rho z-\varepsilon$ and $s=\eta(\rho z-\varepsilon) x-\varepsilon_{1}, Q_{2}$ in (50) can be rewritten as follows:

$$
\begin{aligned}
Q_{2}= & \sum_{i_{1}, i_{2} \in\{1,2\}} d_{i_{1}}^{\alpha} \int_{\frac{\varepsilon}{\rho}}^{\infty} \int_{\frac{\varepsilon_{1}}{\eta(\rho z-\varepsilon)}}^{\frac{\varepsilon_{1}+\varepsilon_{2}\left(1+\varepsilon_{1}\right)}{\eta(\rho z-\varepsilon)}} e^{-\frac{d_{i_{2} \varepsilon_{2}\left(1+\varepsilon_{1}\right) x}(\rho z-\varepsilon) x-\varepsilon_{1}}{\eta\left(\rho z i_{1} x\right.} d x} \\
& \times d^{\alpha} e^{-d^{\alpha} z} d z \\
= & \sum_{i_{1}, i_{2} \in\{1,2\}} \frac{d^{\alpha} d_{i_{1}}^{\alpha} e^{-\frac{d^{\alpha} \varepsilon}{\rho}}}{\eta \rho} \int_{0}^{\varepsilon_{2}\left(1+\varepsilon_{1}\right)} \\
\times & \underbrace{\int_{0}^{\infty} \frac{\exp \left(-\frac{d_{i_{2}}^{\alpha} \varepsilon_{2}\left(1+\varepsilon_{1}\right)\left(s+\varepsilon_{1}\right)+d_{i_{1}}^{\alpha} s\left(s+\varepsilon_{1}\right)}{\eta s t}-\frac{d^{\alpha} t}{\rho}\right) d t}{t} d s .}_{Q_{3}}
\end{aligned}
$$

By applying $\mathrm{K}_{v}(z)=\frac{1}{2}\left(\frac{z}{2}\right)^{v} \int_{0}^{\infty} \frac{\exp \left(-t-z^{2} /(4 t)\right)}{t^{v+1}} d t$ in [23, eq. $(8.447)]$, the above integral $Q_{3}$ can be evaluated as follows:

$Q_{3}=2 K_{0}\left(2 \sqrt{\frac{d^{\alpha} d_{i_{2}}^{\alpha} \varepsilon_{2}\left(1+\varepsilon_{1}\right)\left(s+\varepsilon_{1}\right)+d^{\alpha} d_{i_{1}}^{\alpha} s\left(s+\varepsilon_{1}\right)}{\eta \rho s}}\right)$.

Substituting (49), (51) and (52) into (47), and using the fact that $\mathrm{P}_{2}^{C, I}=1-\overline{\mathrm{P}}_{2}^{C, I}$, the first part of the theorem is proved.

The Gaussian-Chebyshev quadrature [26] is applied to approximate the integral $\int_{0}^{\varepsilon_{2}\left(1+\varepsilon_{1}\right)} \mathrm{K}_{0}\left(2 \sqrt{\frac{g_{i_{1}, i_{2}}(s)}{\rho}}\right) d s$ in (32) as follows:

$$
\begin{aligned}
& \int_{0}^{\varepsilon_{2}\left(1+\varepsilon_{1}\right)} \mathrm{K}_{0}\left(2 \sqrt{\frac{g_{i_{1}, i_{2}}(s)}{\rho}}\right) d s \\
& \approx \frac{\pi \varepsilon_{2}\left(1+\varepsilon_{1}\right)}{2 n} \sum_{i=1}^{n}\left|\sin \frac{2 i-1}{2 n} \pi\right| \mathrm{K}_{0}\left(2 \sqrt{\frac{g_{i_{1}, i_{2}}\left(s_{i}\right)}{\rho}}\right),
\end{aligned}
$$

where $s_{i}=\frac{\varepsilon_{2}\left(1+\varepsilon_{1}\right)}{2}\left(1+\cos \frac{2 i-1}{2 n} \pi\right)$, and $n$ is a complexityaccuracy tradeoff parameter.

Recall that the series representation of Bessel function $\mathbf{K}_{0}(z)$ can be expressed as follows [23, eq. (8.447)]:

$$
\begin{aligned}
\mathbf{K}_{0}(z) & =-\ln \left(\frac{z}{2}\right) \sum_{k=0}^{\infty} \frac{\left(\frac{z}{2}\right)^{2 k}}{(k !)^{2}}+\sum_{k=0}^{\infty} \frac{z^{2 k} \Psi(k+1)}{2^{2 k}(k !)^{2}} \\
& \approx-\ln \left(\frac{z}{2}\right) .
\end{aligned}
$$

Based on (44), (53) and (54), $Q_{2}$ in (51) can be approximated as follows:

$$
\begin{aligned}
Q_{2}= & \sum_{i_{1}, i_{2} \in\{1,2\}} \frac{2 d^{\alpha} d_{i_{1}}^{\alpha} e^{-\frac{d^{\alpha} \varepsilon}{\rho}}}{\eta \rho} \int_{0}^{\varepsilon_{2}\left(1+\varepsilon_{1}\right)} \mathrm{K}_{0}\left(2 \sqrt{\frac{g_{i_{1}, i_{2}}(s)}{\rho}}\right) d s \\
\approx & -\sum_{i_{1}, i_{2} \in\{1,2\}} \frac{d^{\alpha} d_{i_{1}}^{\alpha}\left(1-\frac{d^{\alpha} \varepsilon}{\rho}\right)}{\eta \rho} \frac{\pi \varepsilon_{2}\left(1+\varepsilon_{1}\right)}{2 n} \\
& \times \sum_{i=1}^{n}\left|\sin \frac{2 i-1}{2 n} \pi\right| \ln \frac{g_{i_{1}, i_{2}}\left(s_{i}\right)}{\rho} \\
\approx & \sum_{i_{1}, i_{2} \in\{1,2\}} \frac{\pi d^{\alpha} d_{i_{1}}^{\alpha} \varepsilon_{2}\left(1+\varepsilon_{1}\right)}{2 \eta n} \sum_{i=1}^{n}\left|\sin \frac{2 i-1}{2 n} \pi\right| \frac{\ln \rho}{\rho} .
\end{aligned}
$$


When $\rho \rightarrow \infty, Q_{1}$ in (49) can be approximated as follows:

$$
Q_{1} \approx 1-\frac{\tau}{4 \rho} \ln \frac{4 \rho}{\tau} .
$$

According to (55) and (56), the outage probability of $U_{2}$ at high SNR can be approximated as follows:

$$
\begin{aligned}
\mathrm{P}_{2}^{C, I}= & 1-Q_{1}-Q_{2} \\
\approx & \frac{\tau}{4 \rho} \ln \frac{4 \rho}{\tau}-\sum_{i_{1}, i_{2} \in\{1,2\}} \frac{\pi d^{\alpha} d_{i_{1}}^{\alpha} \varepsilon_{2}\left(1+\varepsilon_{1}\right)}{2 \eta n} \\
& \times \sum_{i=1}^{n}\left|\sin \frac{2 i-1}{2 n} \pi\right| \frac{\ln \rho}{\rho} \\
\approx & \frac{\ln \rho}{\rho} .
\end{aligned}
$$

Therefore, the second part of the theorem is proved, and the proof is complete.

\section{APPENDIX C}

\section{PROOF OF THEOREM 3}

Based on (9), (22) and (35), the coverage probability of $U_{1}$ can be expressed as follows:

$$
\begin{aligned}
& \overline{\mathrm{P}}_{1}^{C, I I} \\
& =\underbrace{\operatorname{Pr}\left\{\frac{1}{2} \log _{2}\left(1+\frac{\alpha_{1} \rho_{R}\left|h_{1}\right|^{2}}{\alpha_{2} \rho_{R}\left|h_{1}\right|^{2}+1}\right) \geq R_{1}^{*}, \rho_{R}>\rho_{R, 2}>0\right\}}_{Q_{4}} \\
& +\underbrace{\operatorname{Pr}\left\{\frac{1}{2} \log _{2}\left(1+\rho_{R}\left|h_{1}\right|^{2}\right) \geq R_{1}^{*}, \rho_{R}>0, \rho_{R, 2}=0\right\}}_{Q_{5}} .
\end{aligned}
$$

The first factor in (58), $Q_{4}$, can be expressed as follows:

$$
\begin{aligned}
Q_{4}= & \operatorname{Pr}\left\{1+\rho_{R}\left|h_{1}\right|^{2}>\left(\varepsilon_{1}+1\right)\left(1+\alpha_{2} \rho_{R}\left|h_{1}\right|^{2}\right),\right. \\
& \left.\rho_{R}>\rho_{R, 2}>0\right\} \\
= & \operatorname{Pr}\left\{\frac{\sqrt{1+\rho_{R, 2}\left|h_{2}\right|^{2}}-1}{\left|h_{2}\right|^{2}}<\frac{\rho_{R}\left|h_{1}\right|^{2}-\varepsilon_{1}}{\left(\varepsilon_{1}+1\right)\left|h_{1}\right|^{2}}\right. \\
& \left.\rho_{R}>\rho_{R, 2}>0,\left|h_{1}\right|^{2}>\frac{\varepsilon_{1}}{\rho_{R}}\right\} \\
= & \operatorname{Pr}\left\{\left|h_{2}\right|^{2}>H\left(\left|h_{1}\right|^{2}\right), \rho_{R}>\rho_{R, 2}>0,\left|h_{1}\right|^{2}>\frac{\varepsilon_{1}}{\rho_{R}}\right\},
\end{aligned}
$$

where $H\left(\left|h_{1}\right|^{2}\right)=\frac{\rho_{R, 2}-2 g\left(\left|h_{1}\right|^{2}\right)}{g^{2}\left(\left|h_{1}\right|^{2}\right)}$, and $g\left(\left|h_{1}\right|^{2}\right)=\frac{\rho_{R}\left|h_{1}\right|^{2}-\varepsilon_{1}}{\left(\varepsilon_{1}+1\right)\left|h_{1}\right|^{2}}$.
Recall that the users' channel gains have been ordered as $\left|h_{1}\right|^{2} \leq\left|h_{2}\right|^{2}$. Therefore, $Q_{4}$ in (59) can be further expressed as follows:

$$
\begin{aligned}
Q_{4} & =\underbrace{\operatorname{Pr}\left\{\left|h_{2}\right|^{2}>\left|h_{1}\right|^{2}>K\left(\rho_{R, 2}, \rho_{R}\right)\right\}}_{Q_{41}} \\
& +\underbrace{\operatorname{Pr}\left\{\left|h_{2}\right|^{2}>H\left(\left|h_{1}\right|^{2}\right), \frac{\varepsilon_{1}}{\rho_{R}}<\left|h_{1}\right|^{2}<K\left(\rho_{R, 2}, \rho_{R}\right)\right\}}_{Q_{42}},
\end{aligned}
$$

where $K\left(\rho_{R, 2}, \rho_{R}\right)=\frac{\rho_{R, 2}\left(\varepsilon_{1}+1\right)^{2}-2 \rho_{R}+\sqrt{\Delta\left(\rho_{R, 2}, \rho_{R}\right)}}{2 \rho_{R}^{2}}$, $\Delta\left(\rho_{R, 2}, \rho_{R}\right)=\left(\rho_{R, 2}\left(\varepsilon_{1}+1\right)^{2}-2 \rho_{R}\right)^{2}+4 \rho_{R}^{2}\left(\varepsilon_{1}^{2}+2 \varepsilon_{1}\right)$.
Based on (48), the first factor in (60), $Q_{41}$, can be calculated as follows:

$$
\begin{aligned}
Q_{41}= & \sum_{i_{1}, i_{2} \in\{1,2\}} \int_{\frac{\varepsilon_{2}^{\prime}}{\rho}}^{\infty} \int_{K\left(\rho_{R, 2}, \rho_{R}\right)}^{\infty} \int_{x}^{\infty} d_{i_{2}}^{\alpha} e^{-d_{i_{2}}^{\alpha} y} d y \\
& \times d_{i_{1}}^{\alpha} e^{-d_{i_{1}}^{\alpha} x} d x d^{\alpha} e^{-d^{\alpha} z} d z \\
= & \sum_{i_{1}, i_{2} \in\{1,2\}} \frac{d^{\alpha} d_{i_{1}}^{\alpha}}{d_{i_{1}}^{\alpha}+d_{i_{2}}^{\alpha}} \int_{\frac{\varepsilon_{2}^{\prime}}{\rho}}^{\infty} e^{-\left(d_{i_{1}}^{\alpha}+d_{i_{2}}^{\alpha}\right) K\left(\rho_{R, 2}, \rho_{R}\right)-d^{\alpha} z} d z \\
= & d^{\alpha} \int_{\frac{\varepsilon_{2}^{\prime}}{\rho}}^{\infty} e^{-\left(d_{1}^{\alpha}+d_{2}^{\alpha}\right) K\left(\rho_{R, 2}, \rho_{R}\right)-d^{\alpha} z} d z
\end{aligned}
$$

The second factor in (60), $Q_{42}$, can be calculated by applying (48) as follows:

$$
\begin{aligned}
Q_{42}= & \sum_{\substack{i_{1}, i_{2} \in\{1,2\}\\
}} \int_{\frac{\varepsilon_{2}}{\rho}}^{\infty} \int_{\frac{\varepsilon_{1}}{\rho_{R}}}^{K\left(\rho_{R, 2}, \rho_{R}\right)} \int_{H(x)}^{\infty} d_{i_{2}}^{\alpha} e^{-d_{i_{2}}^{\alpha} y} d y \\
& \times d_{i_{1}}^{\alpha} e^{-d_{i_{1}}^{\alpha} x} d x d^{\alpha} e^{-d^{\alpha} z} d z \\
= & \sum_{\substack{i_{1}, i_{2} \in\{1,2\}\\
}} d_{i_{1}}^{\alpha} \int_{\frac{\varepsilon_{2}^{\prime}}{\rho}}^{\infty} \int_{\frac{\varepsilon_{1}}{\rho_{R}}}^{K\left(\rho_{R, 2}, \rho_{R}\right)} e^{-d_{i_{2}}^{\alpha} H(x)-d_{i_{1}}^{\alpha} x} d x \\
& \times d^{\alpha} e^{-d^{\alpha} z} d z .
\end{aligned}
$$

The second factor in (58), $Q_{5}$, can be evaluated as follows:

$$
\begin{aligned}
Q_{5}= & \left\{\frac{1}{2} \log _{2}\left(1+\rho_{R}\left|h_{1}\right|^{2}\right) \geq R_{1}^{*}, \rho_{R}>0, \rho_{R, 2}=0\right\} \\
= & \left\{\left|h_{1}\right|^{2}>\frac{\varepsilon_{1}}{\eta\left(\rho|h|^{2}-\varepsilon\right)}, \frac{\varepsilon}{\rho}<|h|^{2}<\frac{\varepsilon_{2}^{\prime}}{\rho}\right\} \\
= & \int_{\frac{\varepsilon}{\rho}}^{\frac{\varepsilon_{2}^{\prime}}{\rho}} e^{-\frac{\left(d_{1}^{\alpha}+d_{2}^{\alpha}\right) \varepsilon_{1}}{\eta(\rho z-\varepsilon)}} d^{\alpha} e^{-d^{\alpha} z} d z \\
= & \int_{\frac{\varepsilon}{\rho}}^{\infty} e^{-\frac{\left(d_{1}^{\alpha}+d_{2}^{\alpha}\right) \varepsilon_{1}}{\eta(\rho z-\varepsilon)}} d^{\alpha} e^{-d^{\alpha} z} d z \\
& -\int_{\frac{\varepsilon_{2}^{\prime}}{\rho}}^{\infty} e^{-\frac{\left(d_{1}^{\alpha}+d_{2}^{\alpha}\right) \varepsilon_{1}}{\eta(\rho z-\varepsilon)}} d^{\alpha} e^{-d^{\alpha} z} d z .
\end{aligned}
$$

Similar to (49), the first integral in (63) can be obtained as follows:

$$
\begin{aligned}
& \int_{\frac{\varepsilon}{\rho}}^{\infty} e^{-\frac{\left(d_{1}^{\alpha}+d_{2}^{\alpha}\right) \varepsilon_{1}}{\eta(\rho-\varepsilon)} d^{\alpha} e^{-d^{\alpha} z} d z} \\
& =e^{-\frac{d^{\alpha} \varepsilon}{\rho}} \sqrt{\frac{4 d^{\alpha}\left(d_{1}^{\alpha}+d_{2}^{\alpha}\right) \varepsilon_{1}}{\eta \rho}} \mathbf{K}_{1}\left(\sqrt{\frac{4 d^{\alpha}\left(d_{1}^{\alpha}+d_{2}^{\alpha}\right) \varepsilon_{1}}{\eta \rho}}\right) .
\end{aligned}
$$

Substituting (61)-(63), and (64) into (58), and using the fact that $\mathrm{P}_{1}^{C, I I}=1-\overline{\mathrm{P}}_{1}^{C, I I}$, the first part of the theorem is proved.

Note that $\Delta\left(\rho_{R, 2}, \rho_{R}\right)$ in $K\left(\rho_{R, 2}, \rho_{R}\right)$ can be upper bounded as follows:

$$
\begin{aligned}
\Delta\left(\rho_{R, 2}, \rho_{R}\right) & =\left(\rho_{R, 2}\left(\varepsilon_{1}+1\right)^{2}-2 \rho_{R}\right)^{2}+4 \rho_{R}^{2}\left(\varepsilon_{1}^{2}+2 \varepsilon_{1}\right) \\
& =\left(\varepsilon_{1}+1\right)^{4}\left(\rho_{R, 2}^{2}-\frac{4 \rho_{R, 2} \rho_{R}}{\left(\varepsilon_{1}+1\right)^{2}}+\frac{4 \rho_{R}^{2}}{\left(\varepsilon_{1}+1\right)^{2}}\right) \\
& <\left(\varepsilon_{1}+1\right)^{4}\left(\rho_{R, 2}+\rho_{R}\right)^{2} .
\end{aligned}
$$


Therefore, $K\left(\rho_{R, 2}, \rho_{R}\right)$ can be upper bounded by applying (65) as follows:

$$
\begin{aligned}
K\left(\rho_{R, 2}, \rho_{R}\right) & =\frac{\rho_{R, 2}\left(\varepsilon_{1}+1\right)^{2}-2 \rho_{R}+\sqrt{\Delta\left(\rho_{R, 2}, \rho_{R}\right)}}{2 \rho_{R}^{2}} \\
& <\frac{\rho_{R, 2}\left(\varepsilon_{1}+1\right)^{2}-2 \rho_{R}+\left(\varepsilon_{1}+1\right)^{2}\left(\rho_{R, 2}+\rho_{R}\right)}{2 \rho_{R}^{2}} \\
& <\frac{3\left(\varepsilon_{1}+1\right)^{2}}{2 \rho_{R}}<\frac{c_{1}}{\rho_{R, 2}}
\end{aligned}
$$

where $c_{1}=\frac{3\left(\varepsilon_{1}+1\right)^{2}}{2}$.

Based on (66), $Q_{41}$ in (61) can be lower bounded as follows:

$$
\begin{aligned}
Q_{41} & =d^{\alpha} \int_{\frac{\varepsilon_{2}^{\prime}}{\rho}}^{\infty} e^{-\left(d_{1}^{\alpha}+d_{2}^{\alpha}\right) K\left(\rho_{R, 2}, \rho_{R}\right)-d^{\alpha} z} d z \\
& \geq d^{\alpha} \int_{\frac{\varepsilon_{2}^{\prime}}{\rho}}^{\infty} e^{-\frac{\left(d_{1}^{\alpha}+d_{2}^{\alpha}\right) c_{1}}{\eta\left(\rho z-\varepsilon_{2}^{\prime}\right)}-d^{\alpha} z} d z \\
& =e^{-\frac{d^{\alpha} \varepsilon}{\rho}} \sqrt{\frac{4 d^{\alpha}\left(d_{1}^{\alpha}+d_{2}^{\alpha}\right) c_{1} \varepsilon_{1}}{\eta \rho}} K_{1}\left(\sqrt{\frac{4 d^{\alpha}\left(d_{1}^{\alpha}+d_{2}^{\alpha}\right) c_{1} \varepsilon_{1}}{\eta \rho}}\right) \\
& \approx\left(1-\frac{d^{\alpha} \varepsilon}{\rho}\right)\left(1+\frac{d^{\alpha}\left(d_{1}^{\alpha}+d_{2}^{\alpha}\right) c_{1} \varepsilon_{1}}{\eta \rho} \ln \frac{d^{\alpha}\left(d_{1}^{\alpha}+d_{2}^{\alpha}\right) c_{1} \varepsilon_{1}}{\eta \rho}\right) \\
& \approx 1-\frac{\ln \rho}{\rho} .
\end{aligned}
$$

Since $e^{-\left(d_{i_{1}}^{\alpha}+d_{i_{2}}^{\alpha}\right) \kappa_{1}} \leq 1, Q_{41}$ in (61) can be upper bounded as follows:

$$
\begin{aligned}
Q_{41} & =d^{\alpha} \int_{\frac{\varepsilon_{2}^{\prime}}{\rho}}^{\infty} e^{-\left(d_{1}^{\alpha}+d_{2}^{\alpha}\right) K\left(\rho_{R, 2}, \rho_{R}\right)-d^{\alpha} z} d z \\
& \leq d^{\alpha} \int_{\frac{\varepsilon_{2}^{\prime}}{\rho}}^{\infty} e^{-d^{\alpha} z} d z \\
& =e^{-\frac{d^{\alpha} \varepsilon_{2}^{\prime}}{\rho}} \approx 1-\frac{1}{\rho} .
\end{aligned}
$$

Since $e^{-d_{i_{2}}^{\alpha} H(x)} \leq 1, Q_{42}$ in (62) can be upper bounded as follows:

$$
\begin{aligned}
Q_{42} & \leq \sum_{i_{1}, i_{2} \in\{1,2\}} d_{i_{1}}^{\alpha} \int_{\frac{\varepsilon_{2}^{\prime}}{\rho}}^{\infty} \int_{\frac{\varepsilon_{1}}{\rho_{R}}}^{\kappa_{1}} e^{-d_{i_{1}}^{\alpha} x} d x d^{\alpha} e^{-d^{\alpha} z} d z \\
& =\sum_{i_{1}, i_{2} \in\{1,2\}} \int_{\frac{\varepsilon_{2}^{\prime}}{\rho}}^{\infty}\left(e^{-d_{i_{1}}^{\alpha} \frac{\varepsilon_{1}}{\rho_{R}}}-e^{-d_{i_{1}}^{\alpha} K\left(\rho_{R, 2}, \rho_{R}\right)}\right) d^{\alpha} e^{-d^{\alpha} z} d z .
\end{aligned}
$$

Based on (67) and (68), $Q_{42}$ can be further bounded as follows:

$$
0<Q_{42}<\frac{\ln \rho}{\rho}-\frac{1}{\rho}
$$

Similar to (67), $e^{-\frac{d^{\alpha} \varepsilon}{\rho}} \sqrt{\frac{4 d^{\alpha}\left(d_{1}^{\alpha}+d_{2}^{\alpha}\right) \varepsilon_{1}}{\eta \rho}} \mathbf{K}_{1}\left(\sqrt{\frac{4 d^{\alpha}\left(d_{1}^{\alpha}+d_{2}^{\alpha}\right) \varepsilon_{1}}{\eta \rho}}\right)$ in $Q_{5}$ can be approximated as follows:

$$
\begin{aligned}
& e^{-\frac{d^{\alpha} \varepsilon}{\rho}} \sqrt{\frac{4 d^{\alpha}\left(d_{1}^{\alpha}+d_{2}^{\alpha}\right) \varepsilon_{1}}{\eta \rho}} \mathbf{K}_{1}\left(\sqrt{\frac{4 d^{\alpha}\left(d_{1}^{\alpha}+d_{2}^{\alpha}\right) \varepsilon_{1}}{\eta \rho}}\right) \\
& \approx 1-\frac{\ln \rho}{\rho}
\end{aligned}
$$

Similar to $Q_{41}, \int_{\underline{\varepsilon_{2}}}^{\infty} e^{-\frac{\left(d_{1}^{\alpha}+d_{2}^{\alpha}\right) \varepsilon_{1}}{\eta(\rho z-\varepsilon)}} d^{\alpha} e^{-d^{\alpha} z} d z$ in $Q_{5}$ can be bounded as follows:

$$
1-\frac{\ln \rho}{\rho}<\int_{\frac{\varepsilon_{2}^{\prime}}{\rho}}^{\infty} e^{-\frac{\left(d_{1}^{\alpha}+d_{2}^{\alpha}\right) \varepsilon_{1}}{\eta(\rho z-\varepsilon)}} d^{\alpha} e^{-d^{\alpha} z} d z<1-\frac{1}{\rho} .
$$

Combining (67), (68), (70), (71) with (72), and noting that $\mathrm{P}_{1}^{C, I I}=1-Q_{41}-Q_{42}-Q_{5}$, we have

$$
\mathrm{P}_{1}^{C, I I} \approx \frac{\ln \rho}{\rho} .
$$

Therefore, the second part of the theorem is proved, and the proof is complete.

\section{REFERENCES}

[1] "5G radio access: requirements, concept and technologies," NTT DOCOMO, Inc., Tokyo, Japan, 5G Whitepaper, July 2014.

[2] "Proposed solutions for new radio access," Mobile and wireless communications enablers for the 2020 information society (METIS), Deliverable D.2.4, Feb. 2015.

[3] 3rd Generation Partnership Project (3GPP), "Study on downlink multiuser superposition transmission for LTE," Shanghai, China, Mar. 2015.

[4] Z. Ding, Z. Yang, P. Fan, and H. V. Poor, "On the performance of non-orthogonal multiple access in $5 \mathrm{G}$ systems with randomly deployed users," IEEE Sig. Process. Lett., vol. 21, no. 12, pp. 1501-1505, Dec. 2014.

[5] Y. Saito, A. Benjebbour, Y. Kishiyama, and T. Nakamura, "System level performance evaluation of downlink non-orthogonal multiple access (NOMA)," in Proc. IEEE Annual Symposium on Personal, Indoor and Mobile Radio Communications (PIMRC), London, UK, Sept. 2013.

[6] Z. Yang, Z. Ding, P. Fan, and G. K. Karagiannidis, "On the performance of non-orthogonal multiple access systems with partial channel information," IEEE Trans. Commun., vol. 64, no. 2, pp. 654-667, Feb. 2016.

[7] Y. Liu, Z. Ding, M. Elkashlan, and J. Yuan, "Non-orthogonal multiple access in large-scale underlay cognitive radio networks," IEEE Trans. Veh. Technol., vol. 65, no. 12, pp. $10152-10$ 157, Dec. 2016.

[8] Z. Ding, P. Fan, and H. V. Poor, "Impact of user pairing on $5 \mathrm{G}$ nonorthogonal multiple access," IEEE Trans. Veh. Technol., vol. 65, no. 8, pp. 6010-6023, Aug. 2016.

[9] S. Timotheou and I. Krikidis, "Fairness for non-orthogonal multiple access in 5G systems," IEEE, Sig. Process. Lett., vol. 22, no. 10, pp. 1647-1651, Oct. 2015.

[10] J. Choi, "On the power allocation for a practical multiuser superposition scheme in NOMA systems," IEEE Commun. Letters, vol. 20, no. 3, pp. 438-441, Mar. 2016.

[11] J. Men and J. Ge, "Non-orthogonal multiple access for multiple-antenna relaying networks," IEEE Commun. Letters, vol. 19, no. 10, pp. 16861689, Oct. 2015.

[12] L. R. Varshney, "Transporting information and energy simultaneously," in Proc. IEEE Int. Symp. Inf. Theory (ISIT), Toronto, Canada, Jul. 2008.

[13] X. Zhou, R. Zhang, and C. K. Ho, "Wireless information and power transfer: Architecture design and rate-energy tradeoff," IEEE Trans. Wireless Commun., vol. 61, no. 11, pp. 4754-4767, Nov. 2013.

[14] A. A. Nasir, X. Zhou, S. Durrani, and R. A. Kennedy, "Relaying protocols for wireless energy harvesting and information processing," IEEE Trans. Wireless Commun., vol. 12, no. 7, pp. 3622-3636, Jul. 2013.

[15] Z. Ding, S. M. Perlaza, I. Esnaola, and H. V. Poor, "Power allocation strategies in energy harvesting wireless cooperative networks," IEEE Trans. Wireless Commun., vol. 13, no. 2, pp. 846-860, Feb. 2014.

[16] H. Chen, Y. Li, Y. Jiang, Y. Ma, and B. Vucetic, "Distributed power splitting for SWIPT in relay interference channels using game theory," IEEE Trans. Wireless Commun., vol. 14, no. 1, pp. 410-420, Jan. 2015.

[17] I. Krikidis, "Simultaneous information and energy transfer in large-scale networks with/without relaying," IEEE Trans. Commun., vol. 62, no. 3, pp. 900-912, Mar. 2014.

[18] — "Relay selection in wireless powered cooperative networks with energy storage," IEEE J. Sel. Areas Commun., vol. 33, no. 12, pp. 25962610, Dec. 2015.

[19] K. H. Liu, "Performance analysis of relay selection for cooperative relays based on wireless power transfer with finite energy storage," IEEE Trans. Veh. Technol., vol. 65, no. 7, pp. 5110-5121, Jul. 2016. 
[20] Y. Gu, H. Chen, Y. Li, and B. Vucetic, "Distributed multi-relay selection in accumulate-then-forward energy harvesting relay networks," Available on-line at http://arxiv.org/abs/1602.00339.

[21] Y. Liu, Z. Ding, M. Elkashlan, and H. V. Poor, "Cooperative nonorthogonal multiple access with simultaneous wireless information and power transfer," IEEE J. Sel. Areas Commun., vol. 34, no. 4, pp. 938953, April 2016.

[22] D. N. C. Tse, P. Viswanath, and L. Zheng, "Diversity-multiplexing tradeoff in multiple-access channels," IEEE Trans. Inform. Theory, vol. 50, pp. 1859-1874, Sept. 2004.

[23] I. S. Gradshteyn and I. M. Ryzhik, Table of Integrals, Series and Products, 6th ed. New York: Academic Press, 2000.

[24] J. Hussein, S. S. Ikki, S. Boussakta, and C. C. Tsimenidis, "Performance analysis of opportunistic scheduling in dual-hop multiuser underlay cognitive network in the presence of cochannel interference," IEEE Trans. Veh. Technol., vol. 65, no. 10, pp. 8163-8176, Oct. 2016

[25] H. A. David and H. N. Nagaraja, Order Statistics. John Wiley, New York, 3rd ed., 2003.

[26] E. Hildebrand, Introduction to Numerical Analysis. Dover, New York, USA, 1987.

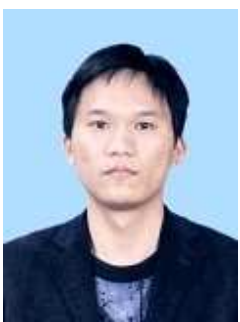

Zheng Yang (S'12) received the B.S. degree in mathematics from Minnan Normal University, Zhangzhou, in 2008, the M.S. degree in mathematics from Fujian Normal University, Fuzhou, China, in 2011, and the Ph.D. degree in information and communications engineering from Southwest Jiaotong University, Chengdu, China, in 2016, respectively.

$\mathrm{He}$ is currently a lecturer at the College of Photonic and Electronic Engineering, Fujian Normal University, China. He was a Visiting Ph.D. Student with the School of Electrical and Electronic Engineering, Newcastle University, Newcastle upon Tyne, U.K., from 2014 to 2014. His research interests include $5 \mathrm{G}$ networks, cooperative and energy harvesting networks, and signal design and coding.

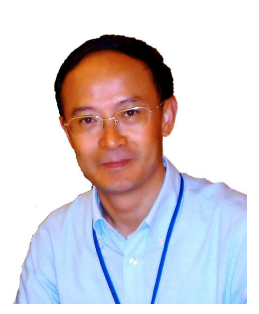

Pingzhi Fan (M'93-SM'99-F'15) received his PhD degree in Electronic Engineering from the Hull University, UK.

$\mathrm{He}$ is currently a professor and director of the institute of mobile communications, Southwest Jiaotong University, China. He is a recipient of the UK ORS Award, the Outstanding Young Scientist Award by NSFC, and the chief scientist of a national 973 research project. He served as general chair or TPC chair of a number of international conferences, and is the guest editor-in-chief, guest editor or editorial member of several international journals. He is the founding chair of IEEE VTS BJ Chapter and IEEE ComSoc CD Chapter, the founding chair of IEEE Chengdu Section. He also served as a board member of IEEE Region 10, IET(IEE) Council and IET Asia-Pacific Region. He has over 200 research papers published in various academic English journals (IEEE/IEE/IEICE, etc), and 8 books (incl. edited), and is the inventor of 22 granted patents.

His research interests include high mobility wireless communications, 5G technologies, wireless networks for big data, signal design and coding, etc. $\mathrm{He}$ is an IEEE VTS Distinguished Lecturer (2015-2017), and a fellow of IEEE, IET, CIE and CIC.

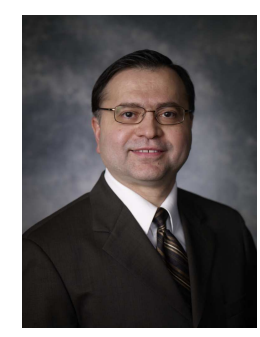

Naofal Al-Dhahir (S'89-M'90-SM'98-F'08) is Erik Jonsson Distinguished Professor at UT-Dallas. He earned his $\mathrm{PhD}$ degree in Electrical Engineering from Stanford University. From 1994 to 2003, he was a principal member of the technical staff at GE Research and AT \& T Shannon Laboratory.

$\mathrm{He}$ is co-inventor of 41 issued US patents, coauthor of over 325 papers with over 7800 citations, and co-recipient of 4 IEEE best paper awards. He is the Editor-in-Chief of IEEE Transactions on Communications and an IEEE Fellow.

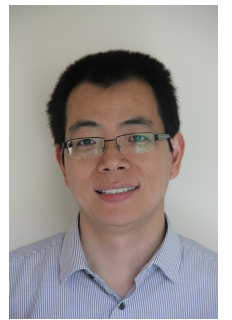

Zhiguo Ding (S'03-M'05-SM'15) received his B.Eng in Electrical Engineering from the Beijing University of Posts and Telecommunications in 2000, and the Ph.D degree in Electrical Engineering from Imperial College London in 2005. From Jul. 2005 to Aug. 2014, he was working in Queen's University Belfast, Imperial College and Newcastle University. Since Sept. 2014, he has been with Lancaster University as a Chair Professor. From Oct. 2012 to Sept. 2016, he has also been an academic visitor in Princeton University.

Dr Ding' research interests are 5G networks, game theory, cooperative and energy harvesting networks and statistical signal processing. He is serving as an Editor for IEEE Transactions on Communications, IEEE Transactions on Vehicular Technology, IEEE Wireless Communications Letters, IEEE Communications Letters, and Journal of Wireless Communications and Mobile Computing. He received the best paper award in IET Comm. Conf. on Wireless, Mobile and Computing, 2009, IEEE Communications Letters Exemplary Reviewer 2012, and the EU Marie Curie Fellowship 2012-2014. 